\title{
Floristic survey of the former royal hunting reserve of Renda, near Palermo (Sicily, Italy)
}

\author{
Marco Giordano', Angelo Troia ${ }^{2 *} \&$ Vincenzo llardi' \\ ${ }^{1}$ Università degli Studi di Palermo, Dipartimento di Scienze della Terra e del Mare (DISTEM), via Archirafi 20, \\ 90123 Palermo, Italy \\ ${ }^{2}$ Università degli Studi di Palermo, Dipartimento di Scienze e Tecnologie Biologiche, Chimiche e Farmaceutiche \\ (STEBICEF), via Archirafi 20, 90123 Palermo, Italy \\ *Corresponding author, email: angelo.troia@unipa.it
}

\begin{abstract}
A mountainous area in western Sicily, where relic wood vegetation is still preserved notwithstanding past and present human pressure, is here analysed in order to prepare a checklist of its vascular flora. Field investigations allowed to compile a floristic inventory including 601 infrageneric taxa belonging to 304 genera and 80 families. Some remarks on the biological and chorological spectra are presented, and some measures to protect flora and vegetation are suggested, too.
\end{abstract}

KEY WORDS Alien species; endemic species; human impact; Mediterranean flora; Palermo Mountains; relic vegetation.

Received 17.03.2021; accepted 11.04.2021; published online 03.06.2021.

\section{INTRODUCTION}

Sicily, the largest island in the Mediterranean, is well-known for its floristic richness connected to its geological history and to its current geographic and geological diversity (Brullo et al., 1995; Médail \& Quézel, 1997; Guarino \& Pasta, 2018): in detail, its flora counts approximately 3250 native species (Guarino \& Pasta, 2018).

We focussed our attention to an area located in the Palermo Mountains, SW of the hamlet of Giacalone (municipality of Monreale). This area, once part of the large territory granted in the XII century by the King William II "the Norman" to the Church of Monreale (Lo Giudice, 1849; Johns, 1981), in the XIX century came in part under the control of the House of Bourbons, who made it a hunting reserve (Sessa, 2015; on the hunting reserves as instrument to return large tracts of land to royal control, see
Hammond, 2013); today it is inserted in the large context of the European "Natura 2000" Ecological Network, according to the 92/43/EEC "Habitats" Directive.

The particular geomorphological and climatic conditions of this area (combined with the indirect protection provided in the XIX century by being the property of the king of the time) allowed the conservation of autochthonous forest formations, especially in the steepest slopes. Today these formations host species of particular phytogeographic interest, although in some cases they have been partially transformed in recent decades into artificial conifer plantations. These particular coenoses are separated by grassland and shrub formations, where the anthropogenic disturbance (e.g., overgrazing, arsons and deforestation) currently occurs.

Given the great naturalistic value of the area, local vascular and moss flora and vegetation was 
already studied by several botanist during the last two centuries: plants collected at 'Renda' or 'Renna' (but also in other sites such as Caculla/Cacudda, Aglisotto, etc., all falling in our study area) are mentioned by famous botanists such as Gussone (1827-1828, 1842-1845), Parlatore (1845, 1848-1896), Lojacono Pojero (1888-1909), etc.; more recently, other researchers (e.g., Marcenò \& Ottonello, 1993; Pasta, 1993; Pasta \& Troia, 1994; Dia et al., 2000) focused their works on this area. The present study aims to supply an updated checklist of local vascular flora, suggesting the most suitable measures for the conservation of an extraordinarily rich biological and environmental heritage.

\section{MATERIAL \& METHODS}

\section{Study area}

The investigated territory, about 714 ha (Fig. 1 ), represents the eastern portion of the Natura 2000 site "Monte Matassaro, Monte Gradara and
Monte Signora" (Code ITA020030), extended about 3,777 ha. The study area is located between $13.197390^{\circ}$ and $13.246755^{\circ}$ of longitude East, and between $38.042293^{\circ}$ and $38.015178^{\circ}$ of latitude North, and its altitude between 675 and $1151 \mathrm{~m}$ a.s.l.

From a geological point of view, the area is located in the Mounts of Palermo, a segment of the Sicilian Apennine range resulting from the tectonic overlap of carbonate and terrigenous-carbonate units of the Mesozoic-Tertiary Age, deriving from the Panormide, Imerese and Trapanese domains, and emplaced after the lower Miocene (Catalano et al., 2000; Catalano et al., 2013). Specifically, the main mountain ranges within the territory, namely Pizzo della Nespola (1086 m a.s.l.), Rocca dell'Aquila (947 m a.s.1.), Pizzo dell'Assolicchiata (1039 m a.s.1.), Monte Matassaro-Renna (1151 m a.s.1.), Cozzo Busino (1012 m a.s.1.), Cozzo Aglisotto (1015 m a.s.1.) and Costalunga (1030 m a.s.l.) (Fig. 2), represent part of the scarp and basin environments of the Imeresi Units, consisting mainly of dolomitic-carbonate, silico-marly and terrigenous deposits. From a morphological point of

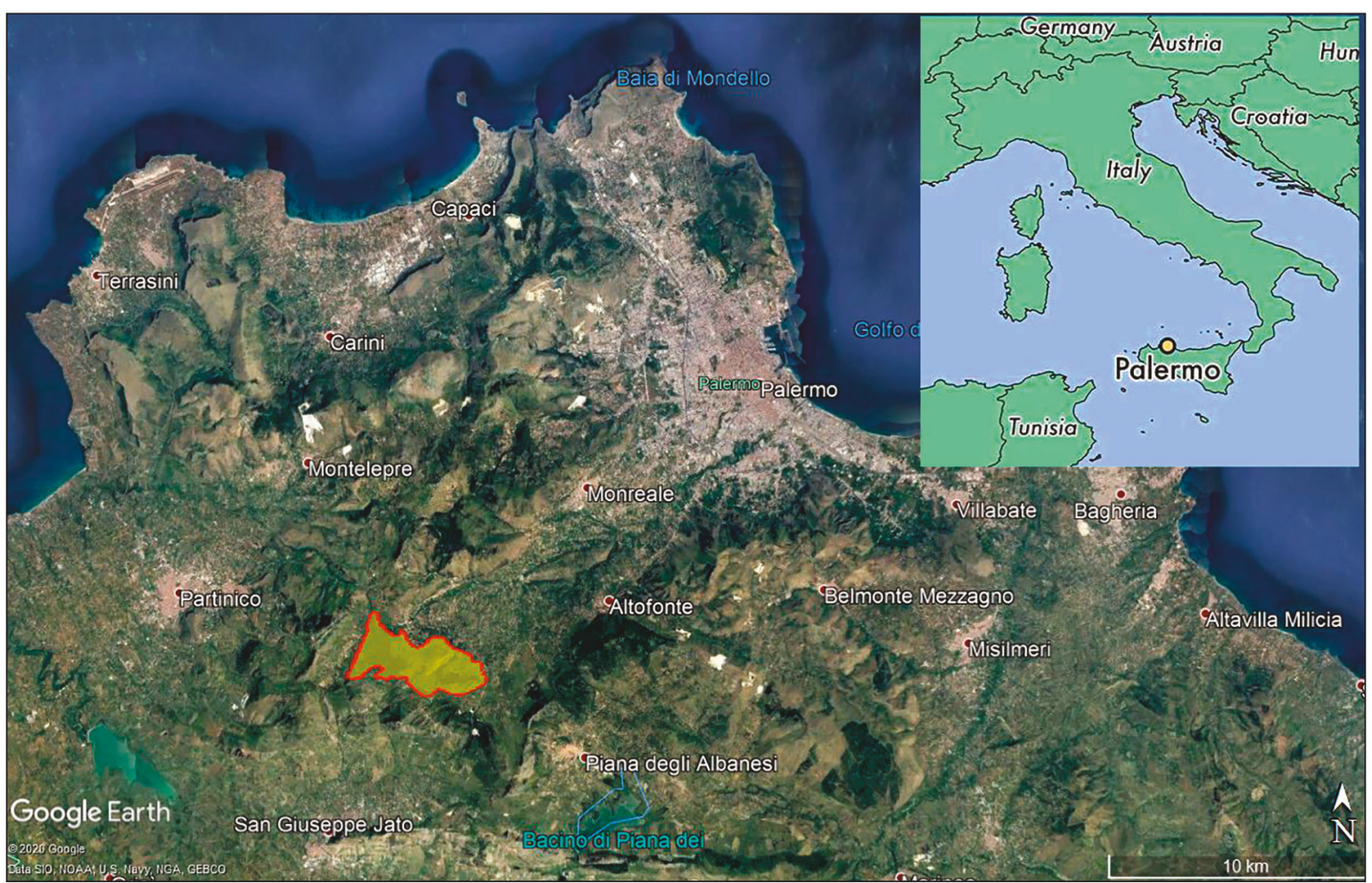

Figure 1. Location of the study area. 
view, these are rocky bodies of considerable size, reliefs with mamelonary shapes and elongated bumps, with slopes that are not too steep, except for those situated in the Northern part, where real vertical cliffs open up.

The characteristics of the mountain context influence the pedology of the area which, according to Fierotti (1997), falls within the association n. 1, "Rock outcrop - Lithic Xerorthents / Rock outcrop - Lithosols", typical to the mountain environments of Sicily.

The particular climatic conditions that can be found here are linked to the particular conformation and position of the mountainous area in question, which make the study area one of the wettest and coldest in the entire province of Palermo (Drago, 2005), with average annual temperature between 13 and $14.5{ }^{\circ} \mathrm{C}$ and average annual rainfall between 1000 and $1100 \mathrm{~mm}$ (without taking into account the importance of the accumulation of dew during the night, with no doubt playing a major role locally; see Dia et al., 2000). Such amount of rain precipitation is remarkable, being equalled only in the highest mountains of the north-eastern sector of the island, like the Nebrodi Mts, the Peloritani Mts and the Etna.

According to Bazan et al. (2015) and in line with the classification introduced by Rivas-Martinez (2004), the study area falls in a "Mediterranean Pluvistagional Oceanic" macrobioclimate, "Upper Mesomediterranean" bioclimate, "lower subhumid" ombrotype. These bioclimatic conditions support the local presence of particular forest communities developing between 800 and $1050 \mathrm{~m}$ a.s.l., referred to the phytosociological syntaxon Aceri campestrisQuercetum ilicis Brullo \& Marcenò 1985 subass. helleboretosum intermedii Marcenò \& Ottonello 1993, endemic to the Palermo Mountains.

\section{Species inventory}

The census of the floristic component was carried out through several field surveys between March 2017 and November 2018, and occasionally during 2019. Plants of dubious identification in the field have been collected, dried and subsequently identified using a binocular microscope and with the help of analytical keys. In particular, the two editions of Pignatti's "Flora d'Italia" have been used (Pignatti, 1982; Pignatti et al., 2017-2019); for

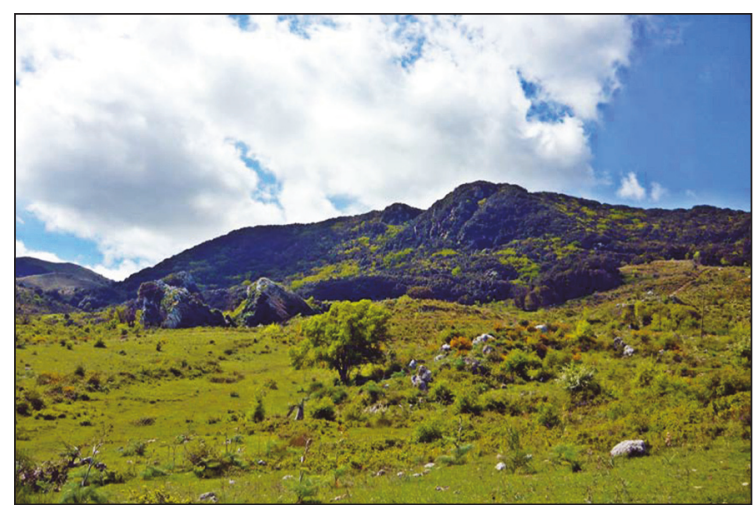

Figure 2. View of the Costalunga wood.

the identification of critical and complex taxa, other Floras and checklists have been consulted (Gussone, 1842-1845; Parlatore, 1848-1896; Lojacono Pojero, 1888-1909; Fiori 1923-1925; Tutin et al., 1964-1980; Greuter et al., 1984-1989).

Original data were compared with historical records issuing from the literature in order to obtain a final list. The list of pteridophytes follows the systematic order suggested by Christenhusz et al. (2011), while the list of angiosperms follows Haston et al. (2009); to make it easier to consult the inventory, the genera and species for each family have been reported in alphabetical order. Species and authors names are in accordance with IPNI (International Plant Name Index, www.ipni.org), while family names are updated according to APW (Angiosperm Phylogeny Website, version 14: http://www.mobot.org/MOBOT/research/APweb/)

The following attributes have also been given for each taxon:

the life form (Raunkiaer, 1934, adapted from Pignatti et al., 2017-2019): phanerophyte (P), nanophanerophyte (NP), chamaephyte $(\mathrm{Ch})$, hemicryptophyte $(\mathrm{H})$, geophyte $(\mathrm{G})$, therophyte $(\mathrm{T})$, helophyte (He);

the chorotype: the chorological categories proposed by Pignatti et al. (2017-2019) were grouped into eight main categories, i.e. Endemic, StenoMediterranean, Euri-Mediterranean, Mediterranean-Mountain, Eurasiatic, Atlantic, Species with wide distribution and Alien species (see Table 1);

as for the status of the listed taxa, its definition (native, naturalized alien, cryptogenic) follows $\mathrm{Ce}-$ lesti-Grapow et al. (2009).

Finally, life form and chorological spectra have 


\begin{tabular}{|c|c|}
\hline Chorological categories used here & Chorological categories according to Pignatti et al. (2017-2019) \\
\hline Steno-Mediterranean & $\begin{array}{l}N-S-E-W-C-S t e n o-M e d i t e r r a n e a n, \text { Steno-Mediterranean- } \\
\text { Macaronesian, Steno-Mediterranean-Pontian, some } N-S-E-W-C- \\
\text { Mediterranean }\end{array}$ \\
\hline Euri-Mediterranean & $\begin{array}{l}N-S-E-W \text {-Euri-Mediterranean, Euri-Medit-Macaronesian, some } \\
N-S-E-W \text {-Mediterranean, a single S-European-Mediterranean }\end{array}$ \\
\hline Mediterranean-Montane & $\begin{array}{l}N-S-E-W \text {-Mediterranean-Montane, (Euri) Mediterranean.- } \\
\text { Montane, a single European-Montane }\end{array}$ \\
\hline Endemic & $\begin{array}{l}\text { Endemic Sicilian, Endemic Sicilian-Apenninic, Endemic, } \\
\text { Subendemic }\end{array}$ \\
\hline Atlantic & $\begin{array}{l}\text { W-Mediterranean-Atlantic, Subatlantic, Submediterranean- } \\
\text { Subatlantic }\end{array}$ \\
\hline Eurasian & $\begin{array}{l}\text { N-S-E-W-Eurasian, Eurasian-Temperate, European-Caucasian, } \\
N \text {-S-E-W-European-Pontian, N-S-E-W-European-Caucasian, } \\
\text { Pontian and Subpontian, European-Subsiberian (Substeppic), SE- } \\
\text { European, Euri-Mediterranean-Pontian, Orophilous-W-Eurasian, } \\
\text { European-W-Asian., Euri-Medit.-S-Sibererian (Subpontian) }\end{array}$ \\
\hline Species with Wide Distribution & $\begin{array}{l}\text { Circumboreal, N-S-E-W-Steno- and Euri-Mediterranean-Turanian, } \\
\text { Cosmopolitan, Subcosmopolitan, Thermo-Cosmop., Palaeo- } \\
\text { Subtropical, Palaeo-Temperate, Saharo-Sindian, Euri- } \\
\text { Mediterranean/C-Asiatiatic (Steppic) }\end{array}$ \\
\hline Alien Species & $\begin{array}{l}\text { N-S-American, S-African, Australian, a single introduced } \\
\text { Steno-Mediterranean (Pinus halepensis) }\end{array}$ \\
\hline
\end{tabular}

Table 1. List of the chorological categories used in this paper, showing the correspondence with categories used by Pignatti et al. (2017-2019).

been produced, and our data compared with those reported by Pignatti (1994) for the entire Sicily. The plants occurring in the study area only under cultivation, deriving from ancient crops or belonging to forest plantations, have been put into a separate table (Table 2), and have not been taken into further consideration in our study, only focused on spontaneous taxa.

\section{RESULTS}

\section{Flora of the area}

Field investigations and species identification led to produce a floristic inventory (Table 3 ) including 601 infrageneric taxa belonging to 304 genera and 80 families (6 Pteridophytes, 1 Gymnosperms and 73 Angiosperms). The most numerous families are Fabaceae (76 taxa), Asteraceae (72 taxa), Poaceae (57 taxa), Apiaceae (28 taxa), Orchideaceae (27 taxa) and Lamiaceae (26 taxa).
The life form spectrum (Fig. 3) highlights the sharp prevalence of therophytes and hemicryptophytes $(36.0 \%$ and $34.6 \%$ respectively), followed by the geophytes $(14.1 \%)$, phanerophytes $(7.3 \%)$, chamaephytes $(5.2 \%)$, nanophanerophytes $(2.5 \%)$ and helophytes (only 2 species, $0.3 \%$ ).

As regards the Chorological Spectrum (Fig. 4), Steno-Mediterranean prevails (34.0\%), followed by the Euri-Mediterranean (20.9\%), Wide distribution taxa (19.1\%), Endemics (9.3\%), Eurasiatic (7.3\%), Mediterranean-Mountain (4.6\%), Atlantic (3.3\%) and Aliens, represented by only $1.3 \%$ of the taxa surveyed.

The autochthonous component amounts to 586 infrageneric taxa, with additional 7 cryptogenic species (Trifolium alexandrinum L., Mespilus germanica L., Acanthus mollis L., Fumaria parviflora Lam., Papaver dubium L., Papaver rhoeas L. and Rhus coriaria L.); 7 allochthonous species are present (Robinia pseudoacacia L., Oxalis pes-caprae L., Euphorbia prostrata Aiton, Eucalyptus camaldulensis Dehnh., Erigeron canadensis L., Symphy- 


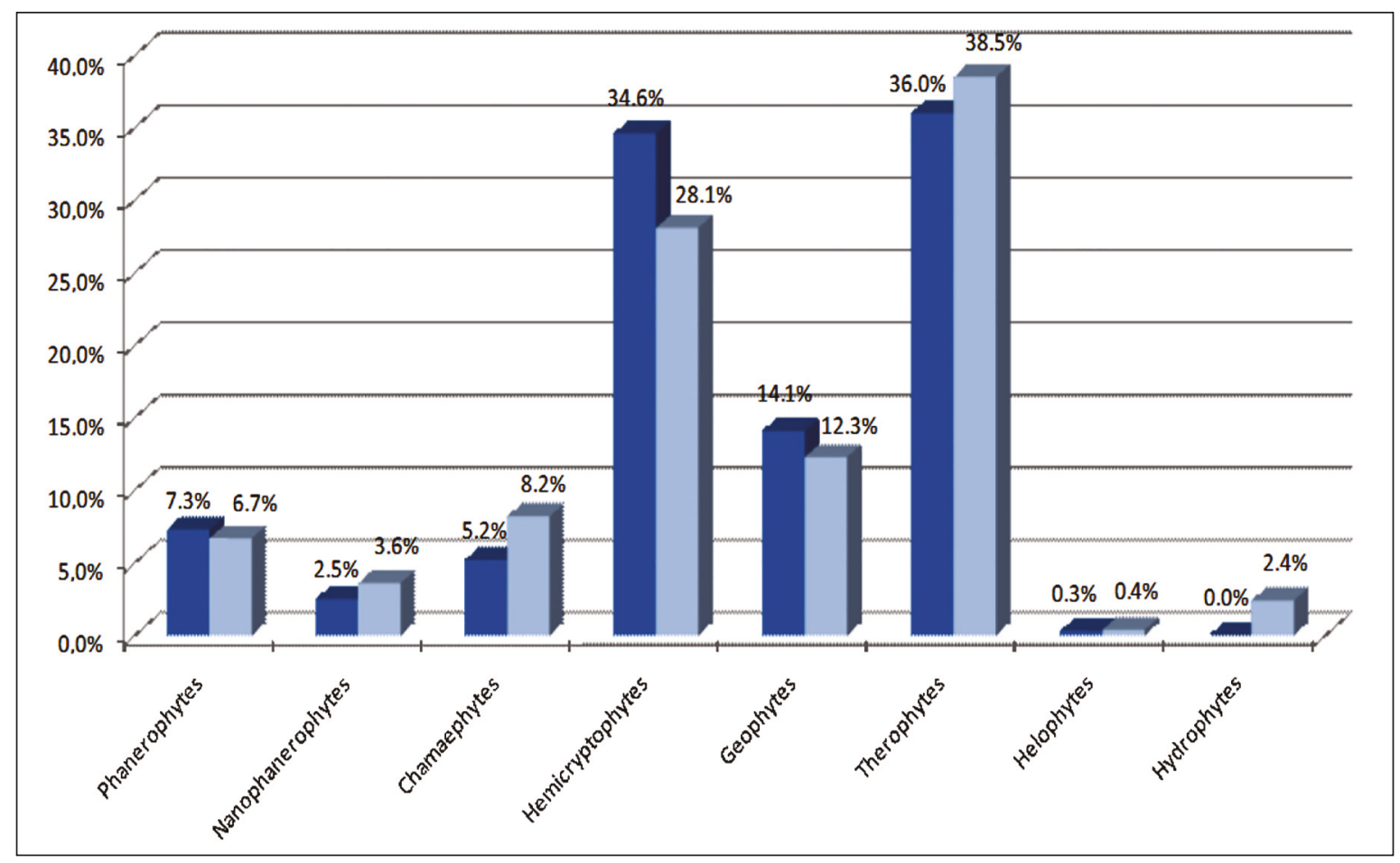

Figure 3. Life form spectrum of the study area (dark columns, on the left) compared to that of the entire Sicily (light columns, on the right) according to Pignatti (1994).

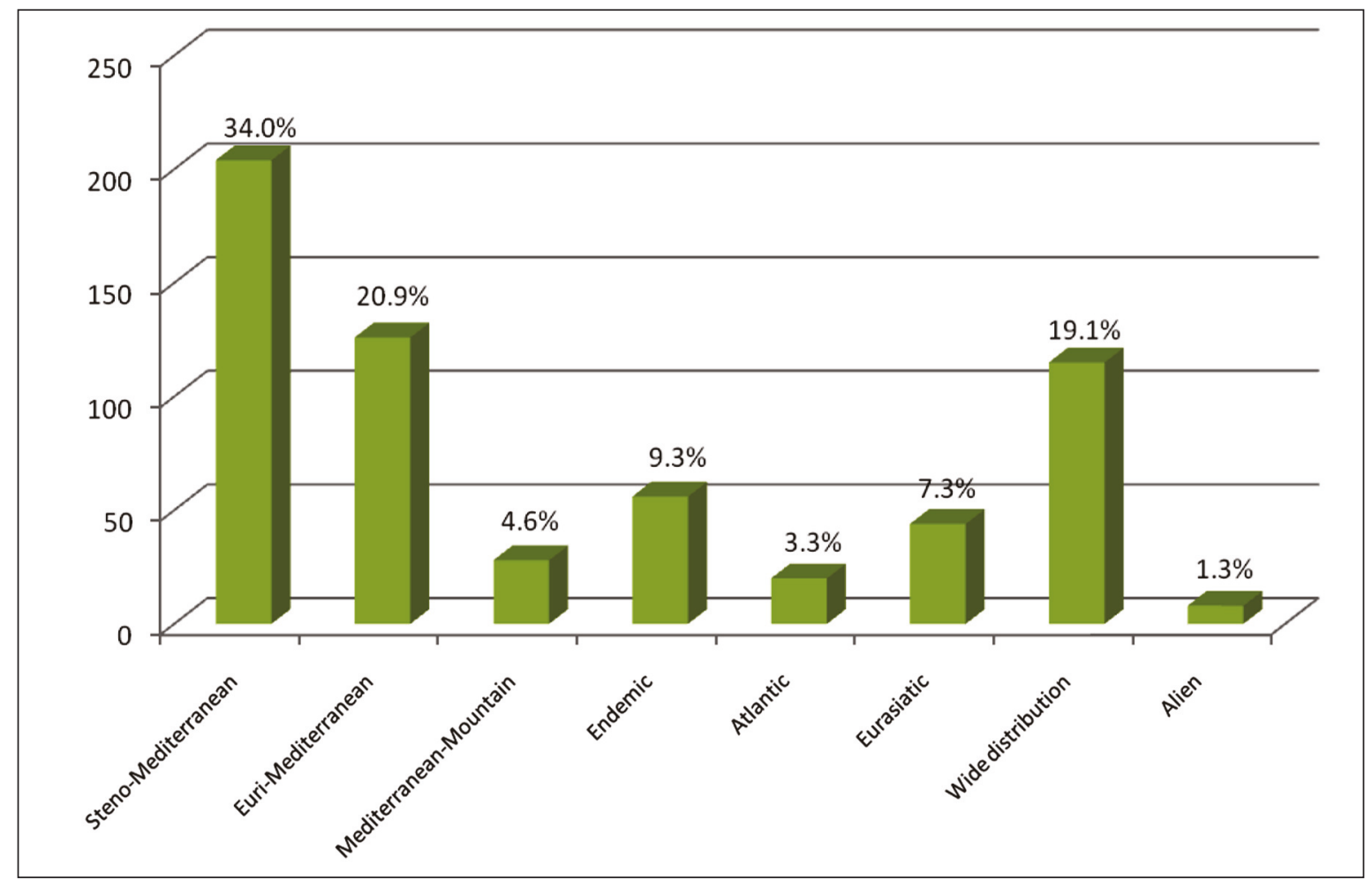

Figure 4. Chorological spectrum of the species found in the study area. See Table 1 for chorological categories. 
otrichum squamatum (Spreng) G. L. Nesom, Xanthium spinosum L.), with an additional eighth species, Pinus halepensis Mill., used for reforestation purposes.

As regards the protection status, only one species, Dianthus rupicola Biv., is reported in Annex II of the "Habitats" Directive, while Mandragora autumnalis Bertol. is present in Annex IV of the aforementioned Directive. Furthermore, 30 taxa (Cyclamen hederifolium Aiton, Cyclamen repandum Sm., Ruscus aculeatus L., and 27 taxa of the Orchidaceae family) are included in CITES within Annex I. The remaining taxa are not subject to any direct form of protection.

\section{Endemic taxa}

In the study area we found a significant number of species endemic to the Palermo Mountains: Brassica rupestris subsp. hispida Raimondo \& P. Mazzola (Fig. 5), a rare mountain taxon whose presence in the study area is unprecedented (Raimondo \& Mazzola, 1997); Allium panormitanum Brullo, Pavone \& Salmeri, element of the thermoxerophilous garrigues and perennial prairie formations linked to Mesozoic limestones (Brullo et al., 2015); Eryngium crinitum C. Presl (Fig. 7), typical of dry grassland environments (Giardina et al., 2007). Another group of species is instead endemic to western Sicily (Drepano-Panormitan district according to Brullo et al., 1995): it includes Anthemis cupaniana Tod. ex Nyman, Centaurea busambarensis Guss., Galium pallidum C. Presl. Finally, the Sicilian endemic species, generally present in the mountainous areas of the island: Ophrys panormitana (Tod.) Soó, Sesleria nitida subsp. sicula Brullo \& Giusso, Trifolium bivonae Guss., Dianthus graminifolius C. Presl, Dianthus siculus C. Presl, Echium italicum subsp. siculum (Lacaita) Greuter \& Burdet, Symphytum gussonei S.W. Schultz, Cymbalaria pubescens (C. Presl) Cufod. (Fig. 6), Odontites bocconei (Guss.) Walp., Crepis bivonana Soldano \& F. Conti and Eryngium tricuspidatum subsp. bocconei (Lam.) A. Wörz.

\section{Species of biogeographical interest}

The presence of species of biogeographical interest is also significant: we verified many species at the southern limit of their range, such as Ilex aquifolium L., Lathyrus venetus (Mill.) Wohlf., Ostrya carpinifolia Scop., Festuca rubra subsp. microphylla St.-Yves, Euphorbia meuselii Geltman [Fig. 8]), but also some North African species that reach the northern limit of their range in Sicily (Ophrys pallida Raf.).

\section{DISCUSSION}

The checklist deriving from our investigations attests a floristically rich area (ca. $18.5 \%$ of the Sicilian flora in an area that is about $0.0003 \%$ of the whole island), with many endemic taxa $(9.3 \%$, versus $6.9 \%$ reported for the whole area of the Palermo Mountains by Gianguzzi et al., 2007) but also with many taxa at the edge of their distribution range.

Regarding the life form spectrum (Fig. 3), the predictable higher incidence of therophytes $(\mathrm{T})$ must be related above all to the Mediterranean climate, characterized by long-lasting hot and dry summers which tend to favour short-cycle species. However, from the comparison with the data provided by Pignatti (1994) for the whole Sicily, a relative lower representativeness of the ephemeral cycle species $(\mathrm{T})$, and a relative greater incidence of herbaceous species with a biennial or perennial cycle $(\mathrm{H})$ and geophytes $(\mathrm{G})$ comes out.

Specifically, the relative great abundance of hemicryptophytes $(\mathrm{H})$ in the study area can be related above all to the particular climatic conditions found here, deriving from the relatively high elevations of the mountains and their arrangement. These observations are further obvious if we compare the data with those relating to the not-far promontory of Monte Pellegrino (600 m a.s.1.) reported by Raimondo et al. (2001); in fact, since in this case it is an isolated carbonate promontory along the Palermo coast, the marked dryness conditions found here tend to favour especially the ephemeral cycle species (T) with an incidence of about $43.0 \%$, to the detriment of the perennial herbaceous species $(\mathrm{H})$, which account for about $23.4 \%$. Similar figures have been reported for the near reserve of Grotta Conza (Gianguzzi et al., 2009), again in the same thermo-mediterranean belt, between 100 and $220 \mathrm{~m}$ a.s.l.

In our study case, on the other hand, the higher altitudes, as well as the morphology of the area, allow the orographic block of the humid air masses 
coming from the Tyrrhenian Sea, resulting in the formation of greater cloudiness in the sky. This determines a lower daily solar radiation, with a consequent reduction in the rate of evapo-transpiration, compared to the lower altitudes, and an additional water supply linked to the fogs, which are very frequent even in the summer. Similar situations (with relative decrease of therophytes and increase of hemicryptophytes) have been reported only for lim- ited areas of the regional territory, such as the Etna, Madonie Mts. and Sicani Mts. (Marcenò et al., 1985).

In addition to the diffusion of grassland communities dominated by perennial species, the above conditions have allowed over time the conservation of some interesting nuclei of forest woodlands, which in such small area present a large number of species, as already mentioned.
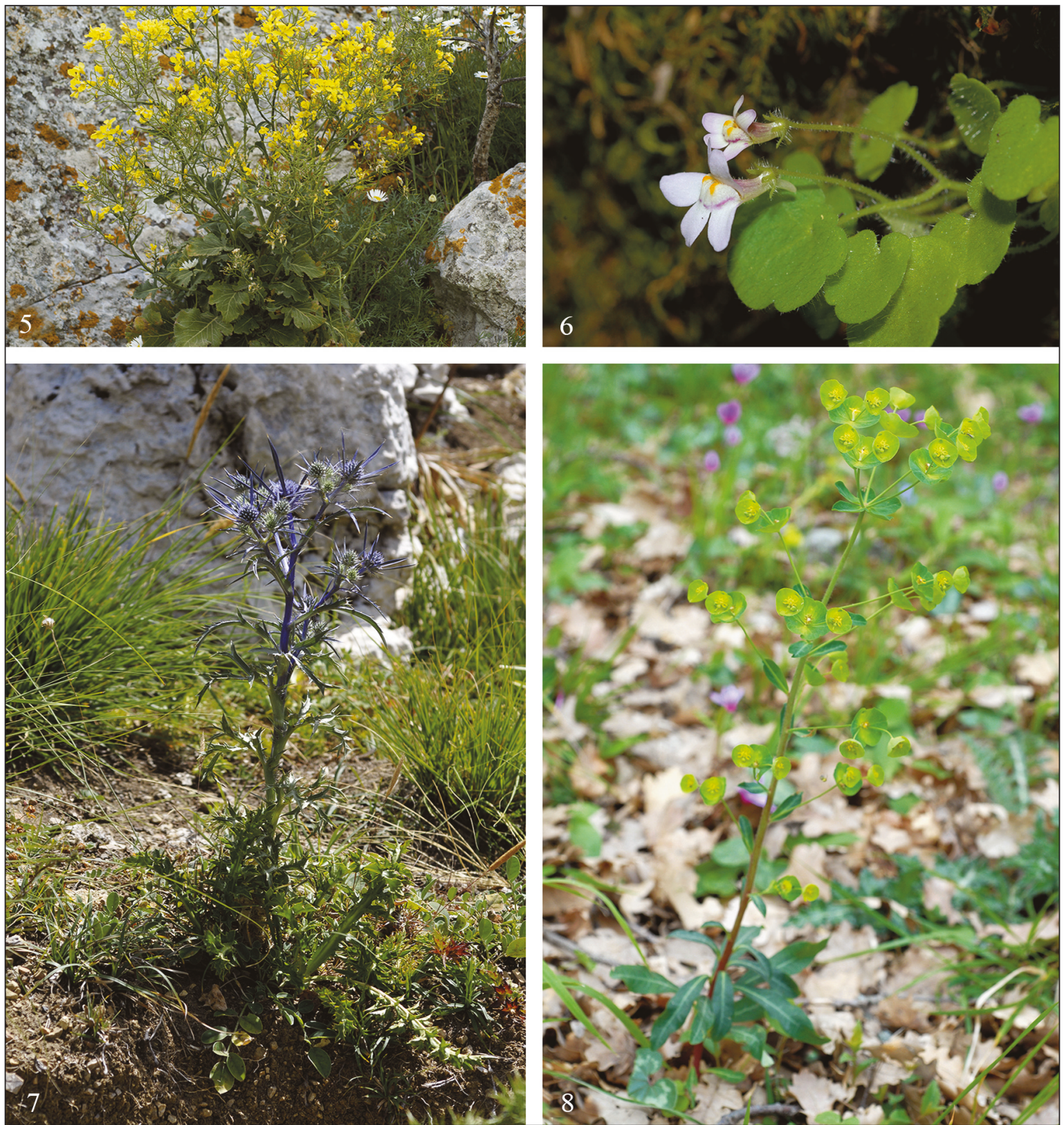

Figures 5-8. Some of the most representative species occurring in the study area (see the text for details). Fig. 5: Brassica rupestris subsp. hispida. Fig. 6: Cymbalaria pubescens. Fig. 7: Eryngium crinitum. Fig. 8: Euphorbia meuselii. 
The investigated area falls, in fact, in an altimetric belt where strictly Mediterranean woody species (Quercus ilex L., Fraxinus ornus L.) can coexist with species of cooler environments [Acer campestris L., Ilex aquifolium L., Ostrya carpinifolia Scop., Cornus sanguinea L., Malus sylvestris (L.) Mill., etc.], gathered in primary forest formations sheltered along the steeper slopes of the reliefs. Not far from these natural woods, artificial forest plantations, dominated by conifers (eventually able to establish and spread naturally like Pinus halepensis), and by Eucalyptus camaldulensis, have been planted.

There are also isolated individuals of species once cultivated (see Table 2), such as the cherry tree (Prunus cerasus L.), the carob (Ceratonia siliqua L.) and the vine (Vitis vinifera L.).

The taxa connected to humid environments are represented only by helophytes (the percentage of which is in line with what is reported for the whole island), namely Carex acutiformis Ehrh. and Typha latifolia L., found on the banks of a small permanent natural pond near the Di Stefano Farmhouse. The lack of hydrophytes can be attributed to the tampering of the natural wetlands, transformed into reservoirs by the fire-fighting service and as such cleaned annually.

The chorological spectrum (Fig. 4) shows the prevalence of Mediterranean species, represented as a whole by the contingents of Endemic, StenoMediterranean, Euri-Mediterranean and Mediterranean-montane species, which all together reach $68.8 \%$ of the surveyed taxa; this result, more than predictable, mostly depends on the geographical position of Sicily within the Mediterranean basin, with its particular climatic characteristics. In particular, the most represented chorotype is that of the StenoMediterranean taxa, which consists of 203 species, with an incidence of $34.0 \%$ [compared to the regional data reported by Pignatti (1994): 29.4\%], a figure which must be related to the presence in the investigated area of open and sunny environments and the presence of lithosols and outcropping rocks. However, the incidence of more temperate taxa is significant, such as the Euri-Mediterranean $(20.8 \%)$, the Mediterranean-montane $(4.6 \%)$ and the Atlantic (3.3\%) elements; the presence of this element $(28.9 \%$ of total incidence) is in line with the altitudinal and morphological characteristics of the mountain ranges, as well as with the particular climatic conditions of the investigated area. The incidence of Eurasian taxa is also important, reaching $7.3 \%$ of the entire floristic contingent surveyed (against 16.8\% reported for the entire region). The spectrum also shows the significant presence of widely distributed species (19.1\%), especially weeds of crops and pastures, which in large part are related to anthropic action, which over time has led to a consequent partial trivialization of the biological heritage.

Nevertheless, the high degree of naturalness that still persists within the investigated area is proven by the presence of a significant number of endemic taxa (56 in total, including 17 Sicilian endemics, 27 Italian endemics and 12 Subendemics), which together account for more than 9\% (precisely 9.3\%) of the local flora, a figure that exceeds the value calculated for the whole Sicily by Pignatti (7.6\%).

Finally, the integrity of these places is further demonstrated by the low incidence of alien species (represented by just $1.3 \%$ of the local flora), which tend to occupy in most cases the most disturbed environments, such as roadsides or the ruderal environments, unable to penetrate into the well-established native formations (with the exception of Eucalyptus camaldulensis which tends to settle within the riparian formations along the Barone-Fontana Fredda Stream). The small amount of alien taxa recorded stands out further if compared, for example, with those calculated by Caldarella et al. (2010) for the not-far Isola delle Femmine reserve, an islet near the coast west of Palermo, where alien species are $9.6 \%$ of the total flora. Consider that the islet includes an extreme environment, where the floristic component is influenced by the harsh morphology, the almost total absence of soil, the marked nitrophilic conditions deriving from the abundant guano released by the colonies of sea birds, as well as from the excessive anthropic exploitation carried out until few years ago: all this certainly facilitated the entry of numerous exotic taxa.

\section{CONCLUSIONS}

This work, regarding the former hunting reserve of Renda, in western Sicily, aims to give the right emphasis (with a quantitative analysis of its flora) to a site that, although it has suffered the effects over 
time of human activities (also considering the relative proximity to the city of Palermo), still preserves a noteworthy naturalistic heritage (Figs. 9-11). Although officially protected as a site of the Natura 2000 network, these habitats continue to suffer direct and indirect anthropogenic disturbance, represented above all by the repeated fires that have occurred during the last ten years, non-regulated overgrazing and overbrowsing and illegal logging (Figs. 12, 13).
These, producing further degradation, will inexorably lead to the complete disappearance and trivialization of the particular floristic component that is preserved here. Therefore, more effective safeguard action should be urgently adopted, also aiming at restoring the near-natural conditions of the site.

To this end, it is important to regulate livestock activities, avoiding the overgrazing and overbrowsing, especially near the wooded areas; in addition,
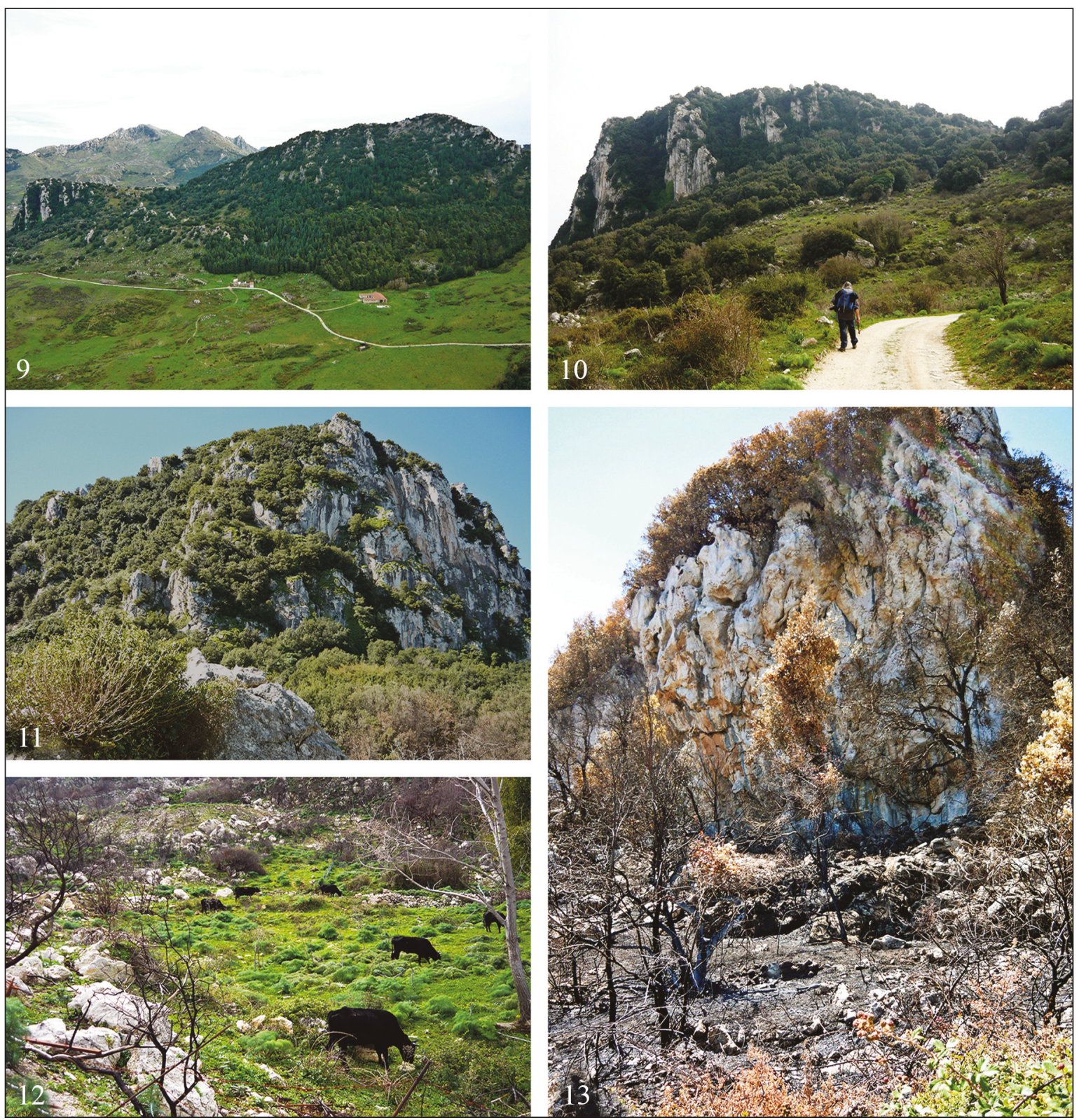

Figures 9-13. Some views of the study area. Fog. 9: view of relict forest formations of Rocca dell'Aquila (on the left) and Pizzo della Nespola (on the right). Figs. 10, 11: details of the vertical oak forest of Rocca dell'Aquila. Fig. 12: grazing cattle near Rocca dell'Aquila e Pizzo della Nespola. Fig. 13: damage caused by the 2017 fire at Costalunga. 
it is important to prevent and fight wildfires, usually linked to human activities.

It is also considered appropriate, in order to favour local progressive succession processes, to plan and carry out the progressive replacement of conifer plantations to allow native species to occupy the spaces left free. In this regard it would be recommended to favour the natural and human-mediated dispersal of the seeds of local native woody species. This would enhance and accelerate local vegetation dynamics, the only ones able to bring back in a few decades the reconstitution of a forest surface similar to the original one.

As regards the protection of the riparian formation along the Barone-Fontana Fredda stream, the eradication of invasive alien species is necessary; in particular we refer to the naturalised individuals of Eucalyptus camaldulensis, currently the only allochthonous species able to threaten the structure and the floristic composition of local riverine forest communities (Badalamenti et al., 2018).
It is also considered desirable to include within the delimitation of the "Natura 2000 site" the small permanent pond present at the entrance gate to the site located near the Di Stefano Farmhouse, within an area reforested with Eucalyptus. Given the presence of an interesting belt of aquatic vegetation, and taking into account the rarity of these environments in the investigated area, this site is certainly worthy of protection.

In conclusion, this work provides a useful tool for the knowledge and more conscious management of a naturalistic environmental heritage of extraordinary richness and beauty, which still preserves evidences of immeasurable value.

\section{ACKNOWLEDGEMENTS}

We thank Salvatore Pasta (CNR-IBBR Palermo, Italy) for his critical reading of a preliminary version of this paper.

\begin{tabular}{|c|c|c|}
\hline Taxon & Life-form & Chorological type \\
\hline \multicolumn{3}{|c|}{ Gymnospermae } \\
\hline \multicolumn{3}{|c|}{ Pinaceae } \\
\hline $\begin{array}{l}\text { Cedrus atlantica (Manetti ex Endl.) Carriere, Traite Gen. } \\
\text { Conif.: } 285 \text { (1855) }\end{array}$ & P scap & Asia \\
\hline Pinus nigra J. F. Arnold, in Reise Mariazell: 8 (1785) & P scap & Illir. \\
\hline Pinus pinea L., Sp. Pl.: 1000 (1753) & P scap & Euri-Medit. \\
\hline \multicolumn{3}{|l|}{ Cupressaceae } \\
\hline $\begin{array}{l}\text { Cupressus arizonica Greene in Bull. Torrey Bot. Club 9:64 } \\
\text { (1882) [Callitropsis arizonica (Greene) D.P. Little] }\end{array}$ & P scap & N-America \\
\hline Cupressus macrocarpa Hartw. in J.Hort.Soc. 2:187 (1847) & P scap & N-America \\
\hline Cupressus sempervirens L., Sp. P1.: 1002 (1753) & P scap & E-Euri-Medit. \\
\hline \multicolumn{3}{|c|}{ Angiospermae } \\
\hline \multicolumn{3}{|c|}{ Vitaceae } \\
\hline Vitis vinifera L., Sp. Pl. 1: 202 (1753). & P lian & SE-Europ.-SW-Asiat. \\
\hline \multicolumn{3}{|c|}{ Fabaceae } \\
\hline Ceratonia siliqua L., Sp. Pl. 2: 1026 (1753). & $\mathrm{P}$ caesp/P scap & S-Medit. \\
\hline Gleditsia triacanthos L., Sp. P1. 2: 1056 (1753). & $\mathrm{P}$ scap/P caesp & N-America \\
\hline \multicolumn{3}{|c|}{ Rosaceae } \\
\hline Prunus cerasus L., Sp. Pl.: 474 (1753) & P scap & Pontico (?) \\
\hline \multicolumn{3}{|c|}{ Fagaceae } \\
\hline Castanea sativa Mill., Gard. Dict., ed. 8. n. 1 (1768) & P scap & SE-Europ. \\
\hline \multicolumn{3}{|c|}{$\begin{array}{ll} & \text { Juglandaceae }\end{array}$} \\
\hline Juglans regia L., Sp. Pl.: 997 (1753) & P scap & SW-Asiat. (?) \\
\hline
\end{tabular}

Table 2. List of the cultivated species in the studied area. 
Table 3. List of the native, naturalized and cryptogenic species in the studied area.

\begin{tabular}{|c|c|c|c|}
\hline Taxon & Life-form & Chorological type & $\begin{array}{l}\text { Native/ } \\
\text { alien status }\end{array}$ \\
\hline \multicolumn{4}{|c|}{ Pteridophyta } \\
\hline \multicolumn{4}{|c|}{ Selaginellaceae } \\
\hline Selaginella denticulata (L.) Spring, Flora 21(10): 149 (1838) & Ch rept & Steno-Medit.-Macaron. & Native \\
\hline \multicolumn{4}{|c|}{$\begin{array}{ll}\text { Equisetaceae } \\
\end{array}$} \\
\hline Equisetum ramosissimum Desf., Fl. Atlant. 2: 398 (1799) & G rhiz & Paleo-Temp. & Native \\
\hline \multicolumn{4}{|c|}{$\begin{array}{ll}\text { Hypolepidaceae } \\
\end{array}$} \\
\hline $\begin{array}{l}\text { Pteridium aquilinum (L.) Kuhn, Reisen Ost Afr. [Decken] } \\
\text { 3(3): Bot. } 11 \text { (1879) }\end{array}$ & G rhiz & Cosmopol. & Native \\
\hline \multicolumn{4}{|l|}{$\begin{array}{ll}\text { Aspleniaceae } \\
\end{array}$} \\
\hline Asplenium onopteris L., Sp. Pl. 2: 1081 (1753) & $\mathrm{H}$ ros & Steno-Medit.-Macaron. & Native \\
\hline $\begin{array}{l}\text { Asplenium trichomanes subsp. quadrivalens D.E. Mey., } \\
\text { Ber. Deutsch. Bot. Ges. 74: } 456 \text { (1962) }\end{array}$ & $\mathrm{H}$ ros & Cosmop-Temp. & Native \\
\hline $\begin{array}{l}\text { Ceterach officinarum DC., Fl. Franc. [de Candolle \& } \\
\text { Lamarck], ed. 3. 2: } 566 \text { (1805) subsp. officinarum }\end{array}$ & $\mathrm{H}$ ros & Eurasiat.-Temp. & Native \\
\hline \multicolumn{4}{|l|}{ Dryopteridaceae } \\
\hline $\begin{array}{l}\text { Dryopteris pallida (Bory) Maire \& Petitm., Bull. Soc. Sci. } \\
\text { Nancy 3, 9: } 480 \text { (1908) subsp. pallida }\end{array}$ & G rhiz & Medit.-Mont & Native \\
\hline $\begin{array}{l}\text { Polystichum setiferum (Forssk.) Moore ex Woynar, Mitt. } \\
\text { Naturwiss. Vereins Steiermark 49: } 181 \text { (1913) }\end{array}$ & G rhiz/H ros & Euri-Medit.-Subatl. & Native \\
\hline \multicolumn{4}{|l|}{ Polypodiaceae } \\
\hline Polypodium cambricum L., Sp. Pl. 2: 1086 (1753) & H ros & Euri-Medit. & Native \\
\hline \multicolumn{4}{|c|}{ Spermatophyta } \\
\hline \multicolumn{4}{|c|}{ Gymnospermae } \\
\hline \multicolumn{4}{|c|}{ Pinaceae } \\
\hline Pinus halepensis Mill., Gard. Dict., ed. 8. n. 8 (1768) & P scap & Steno-Medit. & $\begin{array}{l}\text { *Naturalized } \\
\text { alien }\end{array}$ \\
\hline \multicolumn{4}{|c|}{ Angiospermae } \\
\hline \multicolumn{4}{|c|}{ Lauraceae } \\
\hline Laurus nobilis L., Sp. Pl. 1: 369 (1753) & P caesp(P scap) & Steno-Medit. & Native \\
\hline \multicolumn{4}{|c|}{ Araceae } \\
\hline $\begin{array}{l}\text { Arisarum vulgare O.Targ.Tozz., Ann. Mus. Imp. Fis. } \\
\text { Firenze 2(2): } 67 .(1810)\end{array}$ & G bulb & Steno-Medit. & Native \\
\hline Arum italicum Mill., Gard. Dict., ed. 8. n. 2 (1768) & G rhiz & Steno-Medit. & Native \\
\hline $\begin{array}{l}\text { Biarum tenuifolium (L.) Schott in Schott \& Endlicher, } \\
\text { Melet. Bot.: } 17 \text { (1832) }\end{array}$ & G bulb & Steno-Medit. & Native \\
\hline \multicolumn{4}{|l|}{$\begin{array}{ll}\text { Dioscoreaceae } \\
\end{array}$} \\
\hline Tamus communis L., Sp. Pl. 2: 1028 (1753) & G rad & Euri-Medit. & Native \\
\hline \multicolumn{4}{|c|}{ Smilacaceae } \\
\hline Smilax aspera L., Sp. Pl. 2: 1028 (1753) & $\mathrm{NP}$ & Paleo.-Subtrop. & Native \\
\hline \multicolumn{4}{|c|}{ Orchidaceae } \\
\hline $\begin{array}{l}\text { Anacamptis collina (Banks \& Sol. ex Russell) R.M. Bateman } \\
\text { \& Pridgeon \& M. W. Chase, Lindleyana 12(3): } 120 \text { (1997) }\end{array}$ & G bulb & Steno-Medit. & Native \\
\hline $\begin{array}{l}\text { Anacamptis longicornu (Poir.) R. M. Bateman } \\
\text { \& Pridgeon \& M.W. Chase, Lindleyana 12(3): } 120 \text { (1997) }\end{array}$ & G bulb & W-Steno-Medit. & Native \\
\hline $\begin{array}{l}\text { Anacamptis papilionacea (L.) R.M. Bateman \& Pridgeon \& } \\
\text { M.W. Chase, Lindleyana } 12 \text { (3): } 120 \text { (1997) }\end{array}$ & G bulb & Euri-Medit. & Native \\
\hline Anacamptis pyramidalis (L.) Rich., De Orchid. Eur. 33 (1817) & G bulb & Euri-Medit. & Native \\
\hline
\end{tabular}




\begin{tabular}{|c|c|c|c|}
\hline $\begin{array}{l}\text { Cephalanthera damasonium Druce, Ann. Scott. Nat. Hist. } \\
1906,225\end{array}$ & G rhiz & Euri-Medit. & Native \\
\hline $\begin{array}{l}\text { Epipactis microphylla Sw., Kongl. Vetensk. Acad. } \\
\text { Nya Handl. (1800) } 232\end{array}$ & G rhiz & Europ.-Caucas. & Native \\
\hline $\begin{array}{l}\text { Limodorum abortivum (L.) Sw., Nova Acta Regiae Soc. } \\
\text { Sci. Upsal. 6: } 80 \text { (1799) }\end{array}$ & G rhiz & Euri-Medit. & Native \\
\hline $\begin{array}{l}\text { Neotinea lactea (Poir.) R. M. Bateman \& Pridgeon \& } \\
\text { M.W. Chase, Lindleyana 12(3): } 122 \text { (1997) }\end{array}$ & G bulb & Steno-Medit. & Native \\
\hline $\begin{array}{l}\text { Neotinea tridentata (Scop.) R. M. Bateman \& Pridgeon \& } \\
\text { M.W. Chase, Lindleyana 12(3): } 122 \text { (1997) }\end{array}$ & G bulb & Euri-Medit. & Native \\
\hline Ophrys apifera Huds., Fl. Angl. (Hudson) 340 (1762) & G bulb & (Euri) Medit.-Atl. & Native \\
\hline $\begin{array}{l}\text { Ophrys bertolonii Moretti, Giorn. Fis. Chim. Storia Nat. } \\
\text { Med. Arti Dec. 2, 6: } 145 \text { (1823) }\end{array}$ & G bulb & $\begin{array}{l}\text { C-Steno-Medit. } \\
\text { (Subendem.) }\end{array}$ & Native \\
\hline Ophrys bombyliflora Link, in Schrad. Journ. ii. (1799) 325 & G bulb & W-Steno-Medit. & Native \\
\hline Ophrys fusca Link, in Schrad. Journ. ii. (1799) 324, subsp. fusca & G bulb & Medit.-Atl. & Native \\
\hline $\begin{array}{l}\text { Ophrys fusca subsp. iricolor (Desf.) K.Richt., Pl. Eur. 1: } \\
261 \text { (1890) }\end{array}$ & G bulb & Steno-Medit. & Native \\
\hline Ophrys lutea Cav., Icon. [Cavanilles] ii. 46. t. 160, subsp. lutea & G bulb & Steno-Medit. & Native \\
\hline $\begin{array}{l}\text { Ophrys lutea subsp. sicula (Tineo) Soldano, Atti Soc. Ital. } \\
\text { Sci. Nat. Mus. Civ. Stor. Nat. Milano 133(10): } 115 \text { (1993) }\end{array}$ & G bulb & Steno-Medit. & Native \\
\hline $\begin{array}{l}\text { Ophrys oxyrrhynchos Tod., in Imparziale Giorn. Sc. Lett. } \\
\text { Art. (1840) 74; ex eod. Orch. Sic. } 82\end{array}$ & G bulb & Endem. & Native \\
\hline Ophrys pallida Raf., Caratteri 87 (1810) & G bulb & Subendem. & Native \\
\hline $\begin{array}{l}\text { Ophrys panormitana (Tod.) Soó, Acta Bot. Acad. Sci. } \\
\text { Hung. 18(3-4): } 384 \text { (1973) }\end{array}$ & G bulb & Endem. Sic. & Native \\
\hline $\begin{array}{l}\text { Ophrys tenthredinifera Willd., Sp. Pl., ed. } 4 \text { [Willdenow] 4(1): } \\
67 \text { (1805) }\end{array}$ & G bulb & Steno-Medit. & Native \\
\hline Orchis anthropophora All., Fl. Pedem. ii. 148 (1785) & G bulb & Medit.-Atl. & Native \\
\hline Orchis brancifortii Biv., Stirp. Rar. Sicil. i. [11] t. 1. f. 1. (1813) & G bulb & Endem. & Native \\
\hline Orchis italica Poir., Encycl. [J. Lamarck \& al.] 4(2): 600 (1798) & G bulb & Steno-Medit. & Native \\
\hline Serapias cordigera L., Sp. Pl., ed. 2. 2: 1345 (1763) & G bulb & Steno-Medit. & Native \\
\hline Serapias lingua L., Sp. Pl. 2: 950 (1753) & G bulb & W-Steno-Medit. & Native \\
\hline Serapias parviflora Parl., Giorn. Sci. Sicilia 59: 66 (1837) & G bulb & Steno-Medit. & Native \\
\hline $\begin{array}{l}\text { Serapias vomeracea (Burm.f.) Briq., Prodr. Fl. Corse 1: } \\
378 \text { (1910) }\end{array}$ & G bulb & Euri-Medit. & Native \\
\hline \multicolumn{4}{|l|}{$\begin{array}{ll}\text { Iridaceae } & \text { Iris }\end{array}$} \\
\hline Crocus longiflorus Raf., Caratt. 84. t. 19. f. 2. n. 203 (1810) & G bulb & Subendem. & Native \\
\hline Iris foetidissima L., Sp. Pl. 1: 39 (1753) & G rhiz & Euri-Medit. & Native \\
\hline $\begin{array}{l}\text { Iris planifolia (Mill.) T. Durand \& Schinz, Consp. Fl. Afr. } \\
\text { [T.A. Durand \& H. Schinz] 5: } 143 \text { (1894) }\end{array}$ & G bulb & SW-Steno-Medit. & Native \\
\hline Iris pseudopumila Tineo, Cat. Pl. Hort. Panorm. 283. (1827) & G rhiz & Subendem. & Native \\
\hline $\begin{array}{l}\text { Moraea sisyrinchium Ker Gawl., Ann. Bot. [König \& Sims]. } \\
1(2): 241 \text { (1804) }\end{array}$ & G bulb & Steno-Medit. & Native \\
\hline $\begin{array}{l}\text { Romulea bulbocodium (L.) Sebast. \& Mauri, Fl. Roman. } \\
17 \text { (1818) }\end{array}$ & G bulb & Steno-Medit. & Native \\
\hline Romulea columnae Sebast. \& Mauri, Fl. Roman. 18 (1818) & G bulb & Steno-Medit. & Native \\
\hline Gladiolus italicus Mill., Gard. Dict., ed. 8. n. 2 (1768) & G bulb & Euri-Medit. & Native \\
\hline \multicolumn{4}{|l|}{ Asphodelaceae } \\
\hline Asphodeline lutea (L.) Rchb., Fl. Germ. Excurs. 116 (1830) & G rhiz & E-Medit.-Mont. & Native \\
\hline Asphodelus fistulosus L., Sp. Pl. 1: 309 (1753) & $\begin{array}{l}\text { H scap } \\
\text { (H bienn) }\end{array}$ & Paleo-Subtrop. & Native \\
\hline
\end{tabular}




\begin{tabular}{|c|c|c|c|}
\hline Asphodelus ramosus L., Sp. Pl.: 310 (1753) subsp. ramosus & G tub & Steno-Medit. & Native \\
\hline \multicolumn{4}{|c|}{ Amaryllidaceae } \\
\hline Allium amethystinum Tausch, in Syll. Ratlb. ii. 256 (1824) & G bulb & E-Medit.-Mont. & Native \\
\hline Allium ampeloprasum L., Sp. Pl. 1: 294 (1753) & G bulb & Euri-Medit. & Native \\
\hline Allium nigrum L., Sp. Pl., ed. 2. 1: 430 (1762) & G bulb & Steno-Medit. & Native \\
\hline $\begin{array}{l}\text { Allium panormitanum Brullo, Pavone \& Salmeri, Fl. Medit. } \\
25 \text { (Special Issue): } 216 \text { (2015) }\end{array}$ & G bulb & Endem. Sic. & Native \\
\hline Allium roseum L., Sp. Pl. 1: 296 (1753) & G bulb & Steno-Medit. & Native \\
\hline $\begin{array}{l}\text { Allium sphaerocephalon L., Sp. Pl.: } 297 \text { (1753) subsp. } \\
\text { sphaerocephalon }\end{array}$ & G bulb & Paleo-Temp. & Native \\
\hline Allium subhirsutum L., Sp. Pl. 1: 295 (1753) & G bulb & Steno-Medit. & Native \\
\hline Allium triquetrum L., Sp. Pl. 1: 300 (1753) & G bulb & W-Steno-Medit. & Native \\
\hline Allium vineale L., Sp. Pl. 1: 299 (1753) & G bulb & Euri-Medit. & Native \\
\hline $\begin{array}{l}\text { Narcissus obsoletus Steud., Nomencl. Bot. [Steudel], } \\
\text { ed. 2. 2:182 (1841) }\end{array}$ & G bulb & Steno-Medit. & Native \\
\hline Narcissus tazetta L., Sp. Pl. 1: 290 (1753) subsp. tazetta & G bulb & Steno-Medit. & Native \\
\hline $\begin{array}{l}\text { Nectaroscordum siculum (Ucria) Lindl. in Bot. Reg. 22: } \\
\text { t. } 1912 \text { (1836) }\end{array}$ & G bulb & NW-Steno-Medit. & Native \\
\hline \multicolumn{4}{|l|}{$\begin{array}{ll}\text { Asparagaceae } \\
\end{array}$} \\
\hline Asparagus acutifolius L., Sp. Pl. 1: 314 (1753) & NP & Steno-Medit. & Native \\
\hline $\begin{array}{l}\text { Bellevalia dubia (Guss.) Schult. \& Schult.f., Syst. Veg., ed. } 15 \\
\text { bis 7: } 586 \text { (1830) }\end{array}$ & G bulb & Steno-Medit. & Native \\
\hline $\begin{array}{l}\text { Charybdis pancration (Steinh.) Speta, Phyton (Horn) 38(1): } 60 \\
\text { (1998) }\end{array}$ & G bulb & C-W-Steno-Medit. & Native \\
\hline Loncomelos narbonense (L.) Raf., Autik. Bot. 56 (1840) & G bulb & Euri-Medit. & Native \\
\hline Muscari commutatum Guss., Pl. Rar. 145 (1826) & G bulb & C-Steno-Medit. & Native \\
\hline $\begin{array}{l}\text { Ornithogalum collinum Guss., Index Seminum [Boccadifalco] } \\
\text { 1825: } 9 \text { (1825) }\end{array}$ & G bulb & N-Steno-Medit. & Native \\
\hline Ornithogalum gussonei Ten., Fl. Napol. 3: 371 (1829) & G bulb & Steno-Medit. & Native \\
\hline $\begin{array}{l}\text { Ornithogalum montanum Cirillo in Ten., Fl. Napol. 1: } 176 \\
\text { (1814) }\end{array}$ & G bulb & NE-Medit.-Mont. & Native \\
\hline Ruscus aculeatus L., Sp. Pl. 2: 1041 (1753) & G rhiz/Ch frut & Euri-Medit. & Native \\
\hline \multicolumn{4}{|c|}{ Typhaceae } \\
\hline Typha angustifolia L., Sp. P1. 2: 971 (1753) & $\mathrm{He} / \mathrm{G}$ rhiz & Circumbor. & Native \\
\hline \multicolumn{4}{|c|}{ Juncaceae } \\
\hline Juncus articulatus L., Sp. Pl. 1: 327 (1753) & G rhiz & Circumbor. & Native \\
\hline $\begin{array}{l}\text { Juncus fontanesii J.Gay ex Laharpe, Mém. Soc. Hist. Nat. } \\
\text { Paris iii. (1827) } 130 .\end{array}$ & G rhiz & Paleo-Subtrop. & Native \\
\hline Juncus inflexus L., Sp. Pl. 1: 326 (1753) & H caesp & Paleo-Temp. & Native \\
\hline Juncus subulatus Forssk., Fl. Aegypt.-Arab. 75. (1775) & G rhiz & S-Steno-Medit. & Native \\
\hline Luzula forsteri DC., Syn. Pl. Fl. Gall. 150 (1806) & H caesp & Euri-Medit. & Native \\
\hline \multicolumn{4}{|c|}{\begin{tabular}{|c|} 
Cyperaceae \\
\end{tabular}} \\
\hline Carex acutiformis Ehrh., Beitr. Naturk. [Ehrhart] 4: 43 (1789) & He/G rhiz & Eurasiat. & Native \\
\hline Carex distans L., Syst. Nat., ed. 10. 2: 1263 (1759) & H caesp & Euri-Medit. & Native \\
\hline $\begin{array}{l}\text { Carex flacca Schreb. subsp. serrulata (Biv.) Greuter in } \\
\text { Greuter \& Rech. fil. in Boissiera 13: } 167 \text { (1967) }\end{array}$ & G rhiz & C-Europ.(Steno) & Native \\
\hline Carex halleriana Asso, Syn. Stirp. Aragon. 133 (1779) & H caesp & Euri-Medit. & Native \\
\hline Carex hispida Willd. ex Schkuhr, Beschr. Riedgräs. i. 63 (1801) & G rhiz & Steno-Medit. & Native \\
\hline
\end{tabular}




\section{Poaceae (Graminaceae)}

\begin{tabular}{|c|c|c|c|}
\hline $\begin{array}{l}\text { Ampelodesmos mauritanicus (Poir.) T.Durand \& Schinz, } \\
\text { Consp. Fl. Afr. [T.A. Durand \& H. Schinz] 5: } 874 \text { (1894) }\end{array}$ & H caesp & SW-Steno-Medit. & Native \\
\hline $\begin{array}{l}\text { Anisantha diandra (Roth) Tutin ex Tzvelev, Bot. Mater. } \\
\text { Gerb. Bot. Inst. Komarova Akad. Nauk S.S.S.R. 22: } 4 \text { (1963) }\end{array}$ & T scap & Euri-Medit. & Native \\
\hline $\begin{array}{l}\text { Anisantha madritensis (L.) Nevski, Trudy Sredne-Aziatsk. } \\
\text { Gosud. Univ., Ser. 8b, Bot. 21: (1934), in clavi. }\end{array}$ & T scap & Euri-Medit. & Native \\
\hline $\begin{array}{l}\text { Anisantha rigida (Roth) Hyl., Uppsala Univ. Årsskr. 1945, } \\
\text { no. 7: } 32 \text { (1945) }\end{array}$ & T scap & Paleo-Subtrop. & Native \\
\hline $\begin{array}{l}\text { Anisantha sterilis (L.) Nevski, Trudy Sredne-Aziatsk. } \\
\text { Gosud. Univ., Ser. 8b, Bot. 20: (1934), in clavi. }\end{array}$ & T scap & Euri-Medit.-Turan. & Native \\
\hline Anthoxanthum odoratum L., Sp. P1. 1: 28 (1753) & H caesp & Eurasiat. & Native \\
\hline $\begin{array}{l}\text { Arrhenatherum elatius subsp. bulbosum (Willd.) Schübl. } \\
\text { \& G.Martens, Fl. Wurtemberg (ed. 1) } 70 \text { (1834) }\end{array}$ & H caesp & Paleo-Temp. & Native \\
\hline Avena barbata Pott ex Link, in Schrad. Journ. ii. (1799) 315 & T scap & Euri-Medit.-Turan. & Native \\
\hline Brachypodium retusum P.Beauv., Ess. Agrostogr. 101 (1812) & H caesp & W-Euri-Medit. & Native \\
\hline $\begin{array}{l}\text { Brachypodium rupestre (Host) Roem. \& Schult., Syst. Veg., } \\
\text { ed. } 15 \text { bis [Roemer \& Schultes] 2: } 736 \text { (1817) }\end{array}$ & H caesp & Subatl. & Native \\
\hline Briza maxima L., Sp. Pl. 1: 70 (1753) & T scap & Paleo-Subtrop. & Native \\
\hline $\begin{array}{l}\text { Bromus alopecuros Poir., Voy. Barbarie ii. 100; Vahl, } \\
\text { Symb. Bot. ii. 22. (1789) }\end{array}$ & T scap & Steno-Medit. & Native \\
\hline Bromus hordeaceus L., Sp. Pl. 1: 77 (1753) & T scap & Subcosmop. & Native \\
\hline Bromus intermedius Guss., Fl. Sicul. Prodr. 1: 114 (1827) & T scap & Euri-Medit. & Native \\
\hline Bromus lanceolatus Roth, Catalect. i. 18 (1797) & T scap & Paleo-Temp. & Native \\
\hline Bromus scoparius L., Cent. Pl. I. 6 (1755) & T scap & Steno-Medit. & Native \\
\hline Cynodon dactylon (L.) Pers., Syn. Pl. [Persoon] 1: 85 (1805) & $\mathrm{G}$ rhiz/H rept & Termo-Cosmop. & Native \\
\hline Cynosurus cristatus L., Sp. Pl. 1: 72 (1753) & H caesp/T scap & Europ.-Caucas. & Native \\
\hline Cynosurus echinatus L., Sp. P1. 1: 72 (1753) & T scap & Euri-Medit. & Native \\
\hline Dactylis glomerata L., Sp. Pl. 1: 71 (1753) subsp. glomerata & H caesp & Paleo-Temp. & Native \\
\hline $\begin{array}{l}\text { Dactylis glomerata subsp. hispanica (Roth) Nyman, Consp. } \\
\text { Fl. Eur. } 819 \text { (1882) }\end{array}$ & H caesp & Steno-Medit. & Native \\
\hline $\begin{array}{l}\text { Dasypyrum villosum (L.) P.Candargy, Etude Monogr. Hordees } \\
\text { (Archiv. Biol. Veg. Athenes, Fasc. 1). 35, in clavi, } 62 \text { (1901) }\end{array}$ & T scap & Euri-Medit.-Turan. & Native \\
\hline $\begin{array}{l}\text { Drymochloa drymeja subsp. exaltata (Presl) } \\
\text { Foggi \& Signorini, Willdenowia } 35(2): 242(2005)\end{array}$ & G rhiz & Endem. & Native \\
\hline Festuca ciliata Gouan, Hortus Monsp. 48.547 (1762) & T caesp & Euri-Medit. & Native \\
\hline $\begin{array}{l}\text { Festuca circummediterranea Patzke, Oesterr. Bot. Z. } 122 \text { (4): } \\
261 \text { (1974) }\end{array}$ & H caesp & Euri-Medit. & Native \\
\hline $\begin{array}{l}\text { Festuca geniculata (L.) Lag. et Rodr. Anales Cienc. } 97 \text { Nat. } \\
\text { 6(16): } 150(1803)\end{array}$ & T caesp & W-Steno-Medit. & Native \\
\hline Festuca heterophylla Lam., Fl. Franç. (Lamarck) 3: 600 (1779) & H caesp & Eurasiat. & Native \\
\hline Festuca ligustica Bertol., Amoen. Ital. 8 (1819) & T caesp & Steno-Medit. & Native \\
\hline $\begin{array}{l}\text { Festuca rubra subsp. microphylla St.-Yves, Coste, Monde Pl. } \\
19 \text { (134): } 7 \text { (1922) }\end{array}$ & H caesp & Europ.-Mont. & Native \\
\hline Festuca sicula C.Presl, Cyper. Gram. Sicul. 36. (1820) & H caesp & W-Medit.-Mont. & Native \\
\hline $\begin{array}{l}\text { Gastridium scabrum C.Presl, Fl. Sicul. (Presl) 1: p. xliv, } \\
\text { nomen. (1826) }\end{array}$ & T scap & Steno-Medit. & Native \\
\hline $\begin{array}{l}\text { Gastridium ventricosum (Gouan) Schinz \& Thell., } \\
\text { Vierteljahrsschr. Naturf. Ges. Zürich lviii. } 39 \text { (1913) }\end{array}$ & T scap & Medit.-Atl. & Native \\
\hline Gaudinia fragilis (L.) P.Beauv., Ess. Agrostogr. 95. (1812) & T scap & Euri-Medit. & Native \\
\hline $\begin{array}{l}\text { Helictochloa cincinnata (Ten.) Romero Zarco, } \\
\text { Candollea 66(1): } 102(2011)\end{array}$ & H caesp & SW-Medit.-Mont. & Native \\
\hline
\end{tabular}




\begin{tabular}{|c|c|c|c|}
\hline $\begin{array}{l}\text { Helictotrichon convolutum (C.Presl) Henrard, Blumea iii. } \\
430 \text { (1940). }\end{array}$ & H caesp & NE-Medit.-Mont. & Native \\
\hline Holcus lanatus L., Sp. Pl. 2: 1048 (1753) & H caesp & Circumbor. & Native \\
\hline Hordeum bulbosum L., Cent. Pl. II. 8 (1756) & H caesp & Paleo-Subtrop. & Native \\
\hline $\begin{array}{l}\text { Hordeum murinum subsp. leporinum (Link) Arcang., } \\
\text { Comp. Fl. Ital. [Arcangeli] } 805 \text { (1882) }\end{array}$ & T scap & Circumbor. & Native \\
\hline Lagurus ovatus L., Sp. Pl. 1: 81 (1753) subsp. ovatus & T scap & Euri-Medit. & Native \\
\hline $\begin{array}{l}\text { Lolium multiflorum subsp. gaudinii (Parl.) Schinz \& R. } \\
\text { Keller, Fl. Schweiz ed. 2, 1: } 65 \text { (1905) }\end{array}$ & T scap & Euri-Medit. & Native \\
\hline Lolium perenne L., Sp. Pl.: 83 (1753) & H caesp & Circumbor. & Native \\
\hline $\begin{array}{l}\text { Lolium rigidum Gaudin, Agrost. Helv. i. 334. (1811) subsp. } \\
\text { rigidum }\end{array}$ & T scap & Paleo-Subtrop. & Native \\
\hline Melica ciliata L., Sp. P1. 1: 66 (1753) & H caesp & Euri-Medit.-Turan. & Native \\
\hline $\begin{array}{l}\text { Oloptum miliaceum (L.) Röser \& Hamasha, Pl. Syst. } \\
\text { Evol. 298(2): } 365 \text { (2012) }\end{array}$ & H caesp & Steno-Medit. & Native \\
\hline $\begin{array}{l}\text { Patzkea coerulescens (Desf.) H. Scholz, Willdenowia 40(2): } \\
200 \text { (2010) }\end{array}$ & H caesp & SW-Medit.-Mont. & Native \\
\hline Phalaris coerulescens Desf., Fl. Atlant. 1: 56 (1798) & H caesp & Steno-Medit.-Macaron. & Native \\
\hline $\begin{array}{l}\text { Phleum echinatum Host, Icon. Descr. Gram. Austriac. 3: 8, t. } \\
11 \text { (1805) }\end{array}$ & T scap & NE-Steno-Medit. & Native \\
\hline Poa annua L., Sp. Pl. 1: 68 (1753) & T caesp/ G rhiz & Cosmop. & Native \\
\hline Poa bulbosa L., Sp. Pl. 1: 70 (1753) & H caesp & Subcosmop. & Native \\
\hline Poa sylvicola Guss., Enum. Pl. Inarim. 371 (1855) & H caesp & Euri-Medit. & Native \\
\hline $\begin{array}{l}\text { Polypogon viridis (Gouan) Breistr., Bull. Soc. Bot. France } 110 \\
\text { (Sess. Extraord. 89): } 56 \text { (1966) }\end{array}$ & H caesp & Termo-Cosmop. & Native \\
\hline $\begin{array}{l}\text { Schedonorus arundinaceus Dumort., Observ. Gramin. Belg. } \\
106 \text { (1824) }\end{array}$ & H caesp & Cosmop. & Native \\
\hline $\begin{array}{l}\text { Schedonorus interruptus (Desf.) Tzvelev, Novosti Sist. } \\
\text { Vyssh. Rast. 31: } 259 \text { (1998) subsp. interruptus }\end{array}$ & H caesp & S-Europ.-Medit.(Euri) & Native \\
\hline $\begin{array}{l}\text { Schedonorus pratensis (Huds.) P. Beauv., Ess. Agrostogr. } \\
99,163,177 \text { (1812) subsp. pratensis }\end{array}$ & H caesp & Cosmop. & Native \\
\hline $\begin{array}{l}\text { Sesleria nitida subsp. sicula Brullo \& Giusso, Pl. } \\
\text { Biosystems 140(1): } 45 \text { (-47; figs. 3-4) (2006) }\end{array}$ & H caesp & Endem. Sic. & Native \\
\hline $\begin{array}{l}\text { Trachynia distachyos (L.) Link, Hort. Berol. [Link] 1: } 43 \\
(1827)\end{array}$ & T scap & Steno-Medit.-Turan. & Native \\
\hline $\begin{array}{l}\text { Triticum vagans (Jord. \& Fourr.) Greuter, Boissiera xiii. } 170 \\
\text { (1967) }\end{array}$ & T scap & Steno-Medit.-Turan. & Native \\
\hline \multicolumn{4}{|l|}{$\begin{array}{ll}\text { Papaveraceae } & \text { Pape }\end{array}$} \\
\hline $\begin{array}{l}\text { Fumaria bastardii Boreau, Rev. Bot. Recueil Mens. 2: } \\
359 \text { (1846) }\end{array}$ & T scap & W-Medit.-Atl. & Native \\
\hline Fumaria capreolata L., Sp. Pl. 2: 701 (1753). & T scap & Euri-Medit. & Native \\
\hline $\begin{array}{l}\text { Fumaria flabellata Gasp., Rendiconto Accad. Sci. Soc. } \\
\text { Borbon. Napoli 1: } 51 \text { (1842) }\end{array}$ & T scap & Steno-Medit. & Native \\
\hline $\begin{array}{l}\text { Fumaria parviflora Lam., Encycl. [J. Lamarck \& al.] 2(2): } \\
567 \text { (1788) }\end{array}$ & T scap & $\begin{array}{l}\text { E-Medit.-Turan. } \\
\text { (Archeofita?) }\end{array}$ & Native \\
\hline Papaver dubium L., Sp. Pl. 2: 1196 (1753) & T scap & $\begin{array}{l}\text { E-Medit-Turan. } \\
\text { (Archeofita?) }\end{array}$ & Cryptogenic \\
\hline Papaver rhoeas L., Sp. Pl. 1: 507 (1753) & T scap & $\begin{array}{l}\text { Paleo-Temp. } \\
\text { (Archeofita?) }\end{array}$ & Cryptogenic \\
\hline \multicolumn{4}{|c|}{ Ranunculaceae } \\
\hline $\begin{array}{l}\text { Adonis annua subsp. cupaniana (Guss.) C. H. Steinb., } \\
\text { Webbia } 25 \text { (2): } 324 \text { (1971) }\end{array}$ & T scap & N-Steno-Medit. & Native \\
\hline Anemone coronaria L., Sp. Pl. 1: 539 (1753) & G bulb & $\begin{array}{l}\text { Steno-Medit. } \\
\text { (Archeofita ?) }\end{array}$ & Native \\
\hline
\end{tabular}




\begin{tabular}{|c|c|c|c|}
\hline Anemone hortensis L., Sp. Pl. 1: 540 (1753) & G bulb & N-Steno-Medit. & Native \\
\hline Clematis cirrhosa L., Sp. Pl. 1: 544 (1753) & P lian & Steno-Medit. & Native \\
\hline Clematis vitalba L., Sp. Pl. 1: 543 (1753) & P lian & Europ.-Caucas. & Native \\
\hline Ficaria verna Huds., Fl. Angl. (Hudson) 214 (1762) subsp. verna & G bulb/H scap & Eurasiat. & Native \\
\hline $\begin{array}{l}\text { Helleborus bocconei subsp. intermedius (Guss.) Greuter } \\
\text { \& Burdet, Willdenowia 19(1): } 44 \text { (1989) }\end{array}$ & G rhiz & Endem. & Native \\
\hline Nigella damascena L., Sp. P1. 2: 584 (1753) & T scap & Euri-Medit. & Native \\
\hline Ranunculus angulatus C. Presl, Delic. Prag. 7 (1822) & T scap & Steno-Medit. & Native \\
\hline $\begin{array}{l}\text { Ranunculus bulbosus subsp. aleae (Willk.) Rouy \& Foucaud, } \\
\text { Fl. France 1: } 106 \text { (1893) }\end{array}$ & H scap & Euri-Medit. & Native \\
\hline Ranunculus bullatus L., Sp. Pl. 1: 550 (1753) & $\mathrm{H}$ ros & Steno-Medit. & Native \\
\hline $\begin{array}{l}\text { Ranunculus neapolitanus Ten., Ind. Sem. Hort. Neap. (1825) } \\
\text { 11. n. } 22\end{array}$ & H scap & NE-Steno-Medit. & Native \\
\hline Ranunculus lanuginosus L., Sp. Pl. 1: 554 (1753) & H scap & Europ.-Caucas. & Native \\
\hline $\begin{array}{l}\text { Ranunculus lateriflorus DC., Syst. Nat. [Candolle] 1: } 251 \\
(1817)\end{array}$ & T scap & Paleo-Trop. & Native \\
\hline Ranunculus millefoliatus Vahl, Symb. Bot. (Vahl) ii. 63. (1791) & H scap & Medit.-Mont. & Native \\
\hline Ranunculus muricatus L., Sp. P1. 1: 555 (1753) & T scap & Euri-Medit. & Native \\
\hline Ranunculus pratensis C. Presl, Delic. Prag. 9 (1822) & H scap & Endem. & Native \\
\hline Ranunculus rupestris Guss., Ind. Sem. Boccad. (1826) 8 & H scap & SW-Medit.-Mont. & Native \\
\hline $\begin{array}{l}\text { Thalictrum calabricum Spreng., Pl. Min. Cogn. Pug. } \\
\text { 1: } 37 \text { (1813) }\end{array}$ & H scap & Endem. Sic.-Appenn. & Native \\
\hline \multicolumn{4}{|l|}{$\begin{array}{ll}\text { Paeoniaceae } & \text { Pack } \\
& \text { Pas }\end{array}$} \\
\hline $\begin{array}{l}\text { Paeonia mascula subsp. russoi (Biv.) Cullen \& Heywood, } \\
\text { Feddes Repert. Spec. Nov. Regni Veg. 69: } 35 \text { (1964) }\end{array}$ & G rhiz & Subendem.(C-Medit.) & Native \\
\hline \multicolumn{4}{|l|}{$\begin{array}{ll}\text { Saxifragaceae } & \text { Sa } \\
\end{array}$} \\
\hline Saxifraga bulbifera L., Sp. Pl. 1: 403 (1753) & H scap & NE-Euri-Medit. & Native \\
\hline Saxifraga hederacea L., Sp. Pl. 1: 405 (1753) & T rept & E-Steno-Medit. & Native \\
\hline Saxifraga tridactylites L., Sp. P1. 1: 404 (1753) & T scap & Euri-Medit. & Native \\
\hline \multicolumn{4}{|c|}{ Crassulaceae } \\
\hline $\begin{array}{l}\text { Phedimus stellatus (L.) Raf., Amer. Monthly Mag. \& Crit. } \\
\text { Rev. 1: } 438 \text { (1817). }\end{array}$ & T scap & Steno-Medit. & Native \\
\hline $\begin{array}{l}\text { Sedum album subsp. micranthum (Bast. ex DC.) Syme, } \\
\text { in Sowerby, Engl. Bot. ed. } 3 \text { [B], 4: } 53 \text { (1865) }\end{array}$ & Ch succ & C-Steno-Medit. & Native \\
\hline $\begin{array}{l}\text { Sedum amplexicaule subsp. tenuifolium (Sm.) Greuter, } \\
\text { Willdenowia } 11 \text { (2): } 277 \text { (1981) }\end{array}$ & Ch succ & Steno-Medit. & Native \\
\hline Sedum caeruleum L., Mant. Pl. Altera 241 (1771). & T scap & SW-Steno-Medit. & Native \\
\hline $\begin{array}{l}\text { Sedum caespitosum DC., Prodr. [A. P. de Candolle] } \\
\text { 3: } 405 \text { (1828) }\end{array}$ & T scap & Steno-Medit. & Native \\
\hline $\begin{array}{l}\text { Sedum dasyphyllum L., Sp. Pl. 1: } 431 \text { (1753) subsp. } \\
\text { dasyphyllum }\end{array}$ & Ch succ & Euri-Medit. & Native \\
\hline $\begin{array}{l}\text { Sedum dasyphyllum subsp. glanduliferum (Guss.) Nyman, } \\
\text { in Consp. Fl. Eur.: 263. (1879) }\end{array}$ & Ch suffr & Euri-Medit. & Native \\
\hline Sedum rubens L., Sp. Pl. 1: 432 (1753) & T scap & Euri-Medit.-Subatl. & Native \\
\hline $\begin{array}{l}\text { Sedum sediforme (Jacq.) Pau, Act. \& Mem. Prim. Congr. } \\
\text { Nat. Espan. 1908, } 246 \text { (1909) }\end{array}$ & Ch suffr & Steno-Medit. & Native \\
\hline $\begin{array}{l}\text { Umbilicus horizontalis DC., Prodr. [A. P. de Candolle] 3: } \\
400 \text { (1828) }\end{array}$ & G bulb & Steno-Medit. & Native \\
\hline
\end{tabular}




\begin{tabular}{|c|c|c|c|}
\hline $\begin{array}{l}\text { Umbilicus rupestris (Salisb.) Dandy, Fl. Gloucestershire } \\
611 \text { (1948) }\end{array}$ & G bulb & Steno-Medit.-Atl. & Native \\
\hline \multicolumn{4}{|l|}{$\begin{array}{ll} & \text { Fabaceae }\end{array}$} \\
\hline Anagyris foetida L., Sp. Pl. 1: 374 (1753) & P caesp & Steno-Medit. & Native \\
\hline $\begin{array}{l}\text { Anthyllis maura Beck, in Ann. K. K. Nathurist. Hofmus. } \\
\text { Wien 11: } 64 \text { (1896) }\end{array}$ & H scap/T scap & SW-Steno -Medit. & Native \\
\hline Astragalus hamosus L., Sp. Pl. 2: 758 (1753) & T scap & Medit.-Turan. & Native \\
\hline $\begin{array}{l}\text { Bituminaria bituminosa (L.) C.H.Stirt., Bothalia } 13 \text { (3-4): } \\
318 \text { (1981): (1981) }\end{array}$ & H scap & Euri-Medit. & Native \\
\hline Cytisus infestus Guss., Fl. Sicul. Prodr. 2: 372 (1828) & P caesp & Steno-Medit. & Native \\
\hline $\begin{array}{l}\text { Coronilla scorpioides W.D.J.Koch, Roehl. Deutschl. Fl. } \\
\text { ed. Mert. \& Koch v. } 201\end{array}$ & T scap & Euri-Medit. & Native \\
\hline $\begin{array}{l}\text { Emerus major subsp. emeroides (Boiss. \& Spruner) Soldano } \\
\text { \& F. Conti, Annot. Checkl. Italian Vasc. Fl. } 18 \text { (2005) }\end{array}$ & NP & E-Steno-Medit.-Pontico & Native \\
\hline $\begin{array}{l}\text { Hippocrepis biflora Spreng., Pl. Min. Cogn. Pug. 2: } \\
73 \text { (1815) }\end{array}$ & T scap & Euri-Medit. & Native \\
\hline $\begin{array}{l}\text { Hymenocarpus circinnatus (L.) Savi, Fl. Pis. 2: } \\
205 \text { (1798) }\end{array}$ & T scap & Steno-Medit. & Native \\
\hline Lathyrus amphicarpos L., Sp. Pl. 2: 729 (1753). & T scap & Steno-Medit. & Native \\
\hline Lathyrus annuus L., Demonstr. Pl. 20 (1753). & T scap & Euri-Medit. & Native \\
\hline Lathyrus aphaca L., Sp. P1. 2: 729 (1753). & T scap & Euri-Medit. & Native \\
\hline Lathyrus cicera L., Sp. Pl. 2: 730 (1753). & T scap & Euri-Medit. & Native \\
\hline Lathyrus clymenum L., Sp. Pl. 2: 732 (1753). & T scap & Steno-Medit. & Native \\
\hline Lathyrus hirsutus L., Sp. Pl. 2: 732 (1753). & T scap & Euri-Medit. & Native \\
\hline $\begin{array}{l}\text { Lathyrus ochrus DC., Fl. Franc. [de Candolle \& } \\
\text { Lamarck], ed. 3. 4: } 578 \text { (1805). }\end{array}$ & T scap & Steno-Medit. & Native \\
\hline \multicolumn{4}{|l|}{ Lathyrus odoratus L., Sp. Pl. 2: 732 (1753) } \\
\hline Lathyrus pratensis L., Sp. Pl. 2: 733 (1753) & H scap & Paleo-Temp. & Native \\
\hline Lathyrus sylvestris L., Sp. Pl. 2: 733 (1753) & H scand & Europ.-Caucas. & Native \\
\hline $\begin{array}{l}\text { Lathyrus venetus (Mill.) Wohlf., in W. D. J. Koch, Syn. } \\
\text { Deut. Schweitz. Fl. ed. 3: } 714 \text { (1892) }\end{array}$ & H scap(G rhiz) & Pontico & Native \\
\hline Lotus edulis L., Sp. P1. 2: 774 (1753) & T scap & Steno-Medit. & Native \\
\hline Lotus ornithopodioides L., Sp. Pl. 2: 775 (1753) & T scap & Steno-Medit. & Native \\
\hline Lotus tetragonolobus L., Sp. Pl. 2: 773 (1753) & T scap & Steno-Medit. & Native \\
\hline Lupinus gussoneanus J.Agardh, Syn. Lupini 5 (1835) & T scap & Steno-Medit. & Native \\
\hline Medicago arabica (L.) Huds., Fl. Angl. (Hudson) 288 (1762) & T scap & Euri-Medit. & Native \\
\hline Medicago disciformis DC., Cat. P1. Horti Monsp. 124 (1813) & T scap & Steno-Medit. & Native \\
\hline $\begin{array}{l}\text { Medicago italica (Mill.) Grande, Bull. Orto Bot. Regia } \\
\text { Univ. Napoli 5: } 230 \text { (1916) subsp. italica. }\end{array}$ & T scap & $\begin{array}{l}\text { W-Steno-Medit.-Maca- } \\
\text { ron. }\end{array}$ & Native \\
\hline $\begin{array}{l}\text { Medicago minima (L.) Bartal., Cat. Piante Siena } 60 \text { (-61) } \\
\text { (1776) }\end{array}$ & T scap & $\begin{array}{l}\text { Euri-Medit./C-Asiat. } \\
\text { (Steppica) }\end{array}$ & Native \\
\hline Medicago orbicularis (L.) Bartal., Cat. Piante Siena 60 (1776) & T scap & Euri-Medit. & Native \\
\hline Medicago polymorpha L., Sp. Pl. 2: 779 (1753). & T scap & Subcosmop. & Native \\
\hline Medicago rigidula (L.) All., Fl. Pedem. 1: 316 (1785). & T scap & Euri-Medit. & Native \\
\hline Medicago turbinata (L.) All. Fl. Pedem. 1: 315.(1785) & T scap & Steno-Medit. & Native \\
\hline Medicago truncatula Gaertn., Fruct. Sem. Pl. ii. 150 & T scap & Steno-Medit. & Native \\
\hline $\begin{array}{l}\text { Melilotus italicus (L.) Lam., Fl. Franç. (Lamarck) 2: } 594 \\
\text { (1778) }\end{array}$ & T scap & Steno-Medit. & Native \\
\hline Melilotus sulcatus Desf., Fl. Atlant. 2: 193 (1799). & T scap & Steno-Medit. & Native \\
\hline Ononis oligophylla Ten., Prod. Fl. Nap. Suppl. ii. 69. & T scap & Endem & Native \\
\hline
\end{tabular}




\begin{tabular}{|c|c|c|c|}
\hline Ononis ornithopodioides L., Sp. Pl. 2: 718 (1753) & T scap & Steno-Medit. & Native \\
\hline Ononis pendula Desf., Fl. Atlant. 2: 147, t. 191 (1798) & T scap & $\begin{array}{l}\text { W-Steno-Medit.- } \\
\text { Macaron. }\end{array}$ & Native \\
\hline Ononis reclinata L., Sp. Pl., ed. 2. 2: 1011 (1763) & T scap & S-Medit.-Turan. & Native \\
\hline $\begin{array}{l}\text { Pisum sativum subsp. elatius (M.Bieb.) Asch. \& Graebn., Syn. } \\
\text { Mitteleur. Fl. [Ascherson \& Graebner]. } 6 \text { (2, Lief. 11): } 1064 \text { (1910) }\end{array}$ & T scap & Paleo-Temp. & Native \\
\hline Robinia pseudoacacia L., Sp. Pl. 2: 722 (1753) & P scap/P caesp & N-America & $\begin{array}{l}\text { Naturalized } \\
\text { alien }\end{array}$ \\
\hline Scorpiurus subvillosus L., Sp. Pl. 2: 745 (1753) & T scap & Euri-Medit. & Native \\
\hline Spartium junceum L., Sp. Pl. 2: 708 (1753) & P caesp & Euri-Medit. & Native \\
\hline Sulla coronaria (L.) Medik., Philos. Bot. 1: 209 (1789) & H scap & W-Steno-Medit. & Native \\
\hline Trifolium alexandrinum L., Cent. Pl. I. 25 (1755) & T scap & E-Medit. & Cryptogenic \\
\hline Trifolium angustifolium L., Sp. Pl. 2: 769 (1753) & T scap & Euri-Medit. & Native \\
\hline Trifolium bivonae Guss., Fl. Sicul. Prodr. 2: 512 (1828) & H scap & Endem. Sic. & Native \\
\hline $\begin{array}{l}\text { Trifolium campestre Schreb., Deutschl. Fl. (Sturm), Abt. I, } \\
\text { Phanerog. Heft } 16 \text { (1804) }\end{array}$ & T scap & W-Paleo-Temp. & Native \\
\hline Trifolium cherleri L., Demonstr. Pl. 21 (1753) & T scap & Euri-Medit. & Native \\
\hline $\begin{array}{l}\text { Trifolium fragiferum subsp. bonannii (C. Presl) Sojak, Novit. } \\
\text { Bot. Delect. Sem. Hort. Bot. Univ. Carol. Prag. 1963: } 50 \text { (1963) }\end{array}$ & H rept & Paleo-Temp. & Native \\
\hline $\begin{array}{l}\text { Trifolium grandiflorum Schreb., Nova Acta Phys.-Med. } \\
\text { Acad. Caes. Leop.-Carol. Nat. Cur. 3: } 477 \text { (1767) }\end{array}$ & T scap & E-Steno-Medit. & Native \\
\hline $\begin{array}{l}\text { Trifolium incarnatum subsp. molinerii (Balb.) Syme in C. } \\
\text { Cattaneo, Notiz. Nat. Civil. Lombardia 1: } 292 \text { (1844) }\end{array}$ & T scap/H bienn & Euri-Medit. & Native \\
\hline Trifolium lappaceum L., Sp. P1. 2: 768 (1753) & T scap & Euri-Medit. & Native \\
\hline $\begin{array}{l}\text { Trifolium nigrescens Viv., Fl. Ital. Fragm.: } 12 \text { (1808) subsp. } \\
\text { nigrescens }\end{array}$ & T scap & Euri-Medit. & Native \\
\hline $\begin{array}{l}\text { Trifolium ochroleucon subsp. roseum (C.Presl) Lassen, } \\
\text { Willdenowia 42(2): } 291 \text { (2012) }\end{array}$ & H caesp. & CE-Steno-Medit. & Native \\
\hline $\begin{array}{l}\text { Trifolium physodes Steven ex M.Bieb., Fl. Taur.-Caucas. 2: } \\
217(1808)\end{array}$ & H scap & Steno-Medit. & Native \\
\hline Trifolium pratense L., Sp. Pl. 2: 768 (1753) subsp. pratense & H scap & Subcosmop. & Native \\
\hline $\begin{array}{l}\text { Trifolium pratense subsp. semipurpureum (Strobl) } \\
\text { Pignatti, Giorn. Bot. Ital. 107(5): } 218 \text { (1973). }\end{array}$ & H scap & Endem. & Native \\
\hline $\begin{array}{l}\text { Trifolium repens subsp. prostratum Nyman, Consp. Fl. } \\
\text { Eur.: } 178 \text { (1878) }\end{array}$ & H rept & Subcosmop. & Native \\
\hline Trifolium repens L., Sp. Pl. 2: 767 (1753) subsp. repens & H rept & Subcosmop. & Native \\
\hline $\begin{array}{l}\text { Trifolium resupinatum L., Sp. Pl. 2: } 771 \text { (1753) subsp. } \\
\text { resupinatum }\end{array}$ & T rept (H rept) & Paleo-Temp. & Native \\
\hline Trifolium scabrum L., Sp. P1. 2: 770 (1753) & T rept/T scap & Euri-Medit. & Native \\
\hline $\begin{array}{l}\text { Trifolium squamosum L., Amoen. Acad., Linnaeus ed. 4: } 105 \\
\text { (1759) }\end{array}$ & T scap & Euri-Medit. & Native \\
\hline Trifolium squarrosum L., Sp. Pl. 2: 768 (1753) & T scap & Euri-Medit. & Native \\
\hline Trifolium stellatum L., Sp. Pl. 2: 769 (1753) & T scap & Euri-Medit. & Native \\
\hline Trifolium tomentosum L., Sp. Pl. 2: 771 (1753) & T rept & Paleo-Temp. & Native \\
\hline Vicia bithynica L., Syst. Nat., ed. 10. 2: 1166 (1759) & T scap & Euri-Medit. & Native \\
\hline Vicia grandiflora Scop., Fl. Carniol., ed. 2. 2: 65 (1772) & H scap & SE-Europ.-Pontico & Native \\
\hline Vicia hybrida L., Sp. Pl. 2: 737 (1753) & T scap & Euri-Medit. & Native \\
\hline Vicia leucantha Biv., Stirp. Rar. Sicil. i. [9] (1813) & T scap & SW-Steno-Medit. & Native \\
\hline Vicia lutea subsp. vestita (Boiss.) Rouy, Fl. France 5:219 (1899) & T scap & Euri-Medit. & Native \\
\hline Vicia narbonensis L., Sp. Pl. 2: 737 (1753) & T scap & Euri-Medit. & Native \\
\hline Vicia ochroleuca Ten., Prod. Fl. Nap. p. xlii & H scap & W-Medit.-Mont. & Native \\
\hline
\end{tabular}




\begin{tabular}{|c|c|c|c|}
\hline Vicia sativa L., Sp. Pl.: 736 (1753) subsp. sativa. & T scap & Medit.-Turan. & Native \\
\hline Vicia sicula Guss., Fl. Sicul. Syn. 2(1): 292 (1844). & T scap & SW-Steno-Medit. & Native \\
\hline $\begin{array}{l}\text { Vicia villosa subsp. varia (Host) Corb., Nouv. Fl. Normandie } \\
181 \text { (1894) }\end{array}$ & $\begin{array}{c}\mathrm{T} \operatorname{scap}(\mathrm{H} \\
\text { bienn })\end{array}$ & Euri-Medit. & Native \\
\hline \multicolumn{4}{|c|}{$\begin{array}{ll}\text { Polygalaceae } \\
\end{array}$} \\
\hline Polygala monspeliaca L., Sp. Pl. 2: 702 (1753) & T scap & Steno-Medit. & Native \\
\hline $\begin{array}{l}\text { Polygala preslii Spreng., Syst. Veg., ed. } 16 \text { [Sprengel] } 5 \\
\text { (Index): } 551 \text { (1828) }\end{array}$ & H scap & Endem. & Native \\
\hline \multicolumn{4}{|l|}{$\begin{array}{ll} & \text { Rosaceae }\end{array}$} \\
\hline $\begin{array}{l}\text { Aremonia agrimonoides (L.) DC., Prodr. [A. P. de Candolle] } \\
\text { 2: } 588 \text { (1825). }\end{array}$ & $\mathrm{H}$ ros & NE-Medit-Mont. & Native \\
\hline $\begin{array}{l}\text { Crataegus laevigata (Poir.) DC., Prodr. [A. P. de Candolle] 2: } \\
630(1825)\end{array}$ & P caesp & Euri-Medit. & Native \\
\hline $\begin{array}{l}\text { Crataegus monogyna Jacq., Fl. Austriac. (Jacquin) 3: } 50 \\
\text { (1775). subsp. monogyna }\end{array}$ & P caesp & Paleo-Temp. & Native \\
\hline $\begin{array}{l}\text { Crataegus orientalis subsp. presliana K.I.Chr., Syst. } \\
\text { Bot. Monogr. 35: } 44 \text { (1992) }\end{array}$ & P caesp & W-Medit-Mont. & Native \\
\hline Malus sylvestris (L.) Mill., Gard. Dict., ed. 8. Malus no. 1 (1768) & P scap & C-Europ.-Caucas. & Native \\
\hline Mespilus germanica L., Sp. Pl. 1: 478 (1753) & P caesp/P scap & S-Europ.-Pontico(?) & Cryptogenic \\
\hline Potentilla recta L., Sp. Pl. 1: 497 (1753) & H scap & Paleo-Temp. & Native \\
\hline Potentilla reptans L., Sp. Pl. 1: 499 (1753) & H scap & Subcosmop. & Native \\
\hline Pyrus pyraster Du Roi, Harbk. Baumz. 2: 215 (1772) & P scap & Europ.-Caucas. & Native \\
\hline Pyrus spinosa Forssk., Fl. Aegypt.-Arab. 211.(1775) & P scap & Steno-Medit. & Native \\
\hline Prunus spinosa L., Sp. Pl. 1: 475 (1753) & $\mathrm{P}$ caesp/NP & Europ.-Caucas. & Native \\
\hline Rosa canina L., Sp. Pl. 1: 492 (1753) & $\mathrm{NP}$ & Paleo-Temp. & Native \\
\hline Rosa sempervirens L., Sp. Pl. 1: 492 (1753) & $\mathrm{P}$ caesp/NP & Steno-Medit. & Native \\
\hline Rubus canescens DC., Cat. Pl. Horti Monsp. 139 (1813) & $\mathrm{NP}$ & NE-Medit. (Euri) & Native \\
\hline $\begin{array}{l}\text { Rubus ulmifolius Schott, Isis oder Encyclopedische Zeitung } \\
1818 \text { (1818) }\end{array}$ & NP & Euri-Medit. & Native \\
\hline $\begin{array}{l}\text { Sanguisorba minor subsp. rupicola (Boiss. \& Reut.) O. Bolòs } \\
\text { \& Vigo, Butl. Inst. Catalana Hist. Nat., Secc. Bot. 38(1): } 67 \text { (1974) }\end{array}$ & H scap & W-Steno-Medit. & Native \\
\hline Sorbus domestica L., Sp. Pl. 1: 477 (1753) & P scap & N-Euri-Medit. & Native \\
\hline Sorbus torminalis (L.) Crantz, Stirp. Austr. Fasc. 2: 45 (1763) & P caesp/P scap & S-Europ.-Caucas. & Native \\
\hline \multicolumn{4}{|l|}{ Rhamnaceae } \\
\hline Rhamnus alaternus L., Sp. Pl. 1: 193 (1753) & $\mathrm{P}$ caesp/P scap & Steno-Medit. & Native \\
\hline \multicolumn{4}{|c|}{$\begin{array}{ll} & \text { Ulmaceae } \\
\end{array}$} \\
\hline Ulmus canescens Melville, Kew Bull. 12(3): 499 (1958) & P caesp/P scap & E-Steno-Medit. & Native \\
\hline \multicolumn{4}{|c|}{ Moraceae } \\
\hline Ficus carica L., Sp. Pl. 2: 1059 (1753) & P scap & Medit.-Turan. & Native \\
\hline \multicolumn{4}{|c|}{ Urticaceae } \\
\hline Parietaria lusitanica L., Sp. Pl. 2: 1052 (1753) & T rept & Steno-Medit. & Native \\
\hline Parietaria judaica L., Fl. Palaest. [C. Linnaeus] 32 (1756) & H scap & Euri-Medit.- Macaron. & Native \\
\hline $\begin{array}{l}\text { Urtica membranacea Poir. ex Savigny, Encycl. [J. Lamarck } \\
\text { \& al.] 4(2): } 638 \text { (1798) }\end{array}$ & T scap & S-Steno-Medit. & Native \\
\hline Urtica pilulifera L., Sp. Pl. 2: 983 (1753) & T scap & S-Euri-Medit. & Native \\
\hline Urtica urens L., Sp. Pl. 2: 984 (1753) & T scap & Subcosmop. & Native \\
\hline \multicolumn{4}{|c|}{ Fagaceae } \\
\hline Quercus ilex L., Sp. Pl. 2: 995 (1753) & P scap & Steno-Medit. & Native \\
\hline
\end{tabular}




\begin{tabular}{|c|c|c|c|}
\hline Quercus virgiliana (Ten.) Ten., Fl. Napol. 5: 262 & P scap & E-Steno-Medit. & Native \\
\hline \multicolumn{4}{|c|}{ Betulaceae } \\
\hline Ostrya carpinifolia Scop., Fl. Carniol., ed. 2. 2: 244 (1772) & P caesp/P scap & Eurasiat. & Native \\
\hline \multicolumn{4}{|c|}{ Celastraceae } \\
\hline Euonymus europaeus L., Sp. Pl. 1: 197 (1753) & $\begin{array}{l}\text { P caesp } \\
\text { (P scap) }\end{array}$ & Eurasiat. & Native \\
\hline \multicolumn{4}{|c|}{ Oxalidaceae } \\
\hline Oxalis pes-caprae L., Sp. Pl. 1: 434 (1753) & G bulb & S-Africa(Neofita) & $\begin{array}{l}\text { Naturalized } \\
\text { alien }\end{array}$ \\
\hline \multicolumn{4}{|c|}{ Euphorbiaceae } \\
\hline Euphorbia ceratocarpa Ten., Fl. Napol. 1: 268, t. 43 & Ch suffr & Endem. & Native \\
\hline Euphorbia characias L., Sp. Pl. 1: 463 (1753) & NP & Steno-Medit. & Native \\
\hline Euphorbia exigua L., Sp. Pl. 1: 456 (1753) & T scap & Euri.Medit. & Native \\
\hline Euphorbia falcata L., Sp. Pl. 1: 456 (1753) & T scap & Euri-Medit.-Turan. & Native \\
\hline Euphorbia helioscopia L., Sp. Pl. 1: 459 (1753) & T scap & Cosmop. & Native \\
\hline Euphorbia meuselii Geltman, Komarovia 2: 24 (2002) (2002) & Ch suffr & Endem. & Native \\
\hline Euphorbia peplus L., Sp. Pl. 1: 456 (1753) & T scap & Cosmop. & Native \\
\hline $\begin{array}{l}\text { Euphorbia prostrata Aiton, Hort. Kew. [W. Aiton] 2: } 139 \\
(1789)\end{array}$ & T rept & N-America & $\begin{array}{l}\text { Naturalized } \\
\text { alien }\end{array}$ \\
\hline Euphorbia rigida M.Bieb., Fl. Taur.-Caucas. 1: 375 (1808) & Ch suffr & S-Europ.-Pontico & Native \\
\hline Mercurialis annua L., Sp. Pl. 2: 1035 (1753) & T scap & Paleo-Temp. & Native \\
\hline Mercurialis perennis L., Sp. Pl. 2: 1035 (1753) & G rhiz & Europ.-Caucas. & Native \\
\hline \multicolumn{4}{|c|}{ Salicaceae } \\
\hline Populus nigra L., Sp. Pl. 2: 1034 (1753) subsp. nigra & P scap & Paleo-Temp. & Native \\
\hline Salix alba L., Sp. P1. 2: 1021 (1753) & P scap & Paleo-Temp. & Native \\
\hline Salix pedicellata Desf., Fl. Atlant. 2: 362 (1799) & $\mathrm{P}$ caesp/P scap & W-Steno-Medit. & Native \\
\hline \multicolumn{4}{|c|}{ Violaceae } \\
\hline $\begin{array}{l}\text { Viola alba subsp. dehnhardtii (Ten.) W. Becker in Ber. Bayer. } \\
\text { Bot. Ges. } 8 \text { (2): } 257 \text { (1902) }\end{array}$ & $\mathrm{H}$ ros & Euri-Medit. & Native \\
\hline \multicolumn{4}{|l|}{ Linaceae } \\
\hline Linum bienne Mill., Gard. Dict., ed. 8. n. 8 (1768) & H bienn/H scap & Euri-Medit.-Subatl. & Native \\
\hline Linum corymbulosum Rchb., Fl. Germ. Excurs. 834 (1832) & T scap & Steno-Medit. & Native \\
\hline Linum strictum L., Sp. Pl. 1: 279 (1753) subsp. strictum & T scap & Steno-Medit. & Native \\
\hline Linum trigynum L., Sp. Pl. 1: 279 (1753) & T scap & Euri-Medit. & Native \\
\hline \multicolumn{4}{|c|}{$\begin{array}{ll}\text { Hypericaceae } \\
\end{array}$} \\
\hline $\begin{array}{l}\text { Hypericum hircinum subsp. majus (Aiton) N. Robson, Bull. } \\
\text { Brit. Mus. (Nat. Hist.), Bot. 12(4): } 310 \text { (1985) }\end{array}$ & NP & E-Steno-Medit. & Native \\
\hline Hypericum perfoliatum L., Syst. Nat., ed. 12. 2: 510 (1767) & H scap & Steno-Medit. & Native \\
\hline $\begin{array}{l}\text { Hypericum perforatum L., Sp. Pl. 2: } 785 \text { (1753) subsp. } \\
\text { perforatum }\end{array}$ & H scap & Subcosmop. & Native \\
\hline \multicolumn{4}{|l|}{ Geraniaceae } \\
\hline Geranium columbinum L., Sp. Pl. 2: 682 (1753) & T scap & $\begin{array}{l}\text { Europ.-Subsiber.(Sub- } \\
\text { steppico) }\end{array}$ & Native \\
\hline Geranium lucidum L., Sp. Pl. 2: 682 (1753) & T scap & Euri-Medit. & Native \\
\hline Geranium molle L., Sp. Pl. 2: 682 (1753) & $\begin{array}{c}\text { T scap }(\mathrm{H} \\
\text { bien/H scap) }\end{array}$ & Subcosmop. & Native \\
\hline Geranium robertianum L., Sp. Pl. 2: 681 (1753) & T scap/H bienn & Subcosmop. & Native \\
\hline
\end{tabular}




\begin{tabular}{|c|c|c|c|}
\hline Geranium purpureum Vill., Fl. Delphin. 72 (1785) & T scap & Euri-Medit. & Native \\
\hline Geranium rotundifolium L., Sp. P1. 2: 683 (1753) & T scap & Paleo-Temp. & Native \\
\hline $\begin{array}{l}\text { Erodium acaule Bech. \& Thell., Repert. Spec. Nov. } \\
\text { Regni Veg. 25: } 215 \text { (1928) }\end{array}$ & $\mathrm{H}$ ros & Medit.-Mont. & Native \\
\hline $\begin{array}{l}\text { Erodium cicutarium L'Hér., Hort. Kew. [W. Aiton] 2: } \\
414 \text { (1789) }\end{array}$ & $\begin{array}{c}\mathrm{T} \operatorname{scap}(\mathrm{T} \\
\text { caesp/H ros) }\end{array}$ & Subcosmop.(Sinantrop.) & Native \\
\hline Erodium malacoides (L.) L'Hér., in Ait. Hort. Kew. ii. 415 (1789). & T scap/H bienn & Steno-Medit.-Macaron. & Native \\
\hline $\begin{array}{l}\text { Erodium moschatum L'Hér. ex Aiton, Hort. Kew. [W. Aiton] } \\
\text { 2: } 414 \text { (1789) }\end{array}$ & T scap/H bienn & Euri-Medit. & Native \\
\hline \multicolumn{4}{|l|}{$\begin{array}{ll} & \text { Lythraceae }\end{array}$} \\
\hline $\begin{array}{l}\text { Lythrum junceum Banks ex Sol., Nat. Hist. Aleppo, ed. } 2 \\
\text { [A. Russell] 2: } 253 \text { (1794). }\end{array}$ & H scap(T scap) & Steno-Medit.-Macaron. & Native \\
\hline \multicolumn{4}{|l|}{ Onagraceae } \\
\hline Epilobium hirsutum L., Sp. Pl. 1: 347 (1753) & H scap & Subcosmop. & Native \\
\hline $\begin{array}{l}\text { Epilobium tetragonum subsp. tournefortii (Michalet) H. Lev. } \\
\text { in Monde P1. 6: } 22 \text { (1896) }\end{array}$ & H scap & Paleo-Temp. & Native \\
\hline \multicolumn{4}{|l|}{ Myrtaceae } \\
\hline $\begin{array}{l}\text { Eucalyptus camaldulensis Dehnh., Cat. Pl. Horti Camald., } \\
\text { ed. } 220 \text { (1832) }\end{array}$ & P scap & Australia & $\begin{array}{l}\text { Naturalized } \\
\text { alien }\end{array}$ \\
\hline \multicolumn{4}{|l|}{ Anacardiaceae } \\
\hline Pistacia terebinthus L., Sp. P1. 2: 1025 (1753) & $\begin{array}{l}\text { P caesp (P } \\
\text { scap) }\end{array}$ & Euri-Medit. & Native \\
\hline Rhus coriaria L., Sp. Pl. 1: 265 (1753) & P caesp & Medit.-Pontico & Cryptogenic \\
\hline \multicolumn{4}{|c|}{ Sapindaceae } \\
\hline Acer campestre L., Sp. Pl.: 1055 (1753) & P scap & $\begin{array}{l}\text { Europ.-Caucas.- } \\
\text { Subpontico }\end{array}$ & Native \\
\hline Acer pseudoplatanus L., Sp. Pl.: 1054 (1753) & P scap & Europ.-Caucas. & Native \\
\hline \multicolumn{4}{|c|}{ Rutaceae } \\
\hline Ruta chalepensis L., Mant. Pl. 69 (1767) & Ch suffr & Steno-Medit. & Native \\
\hline \multicolumn{4}{|c|}{ Malvaceae } \\
\hline Lavatera olbia L., Sp. P1. 2: 690 (1753) & NP caesp & Steno-Medit. & Native \\
\hline Lavatera trimestris L., Sp. P1. 2: 692 (1753) & T scap & Steno-Medit. & Native \\
\hline Malva cretica Cav., Diss. 2, Secunda Diss. Bot. 67 (1786) & T scap & Steno-Medit. & Native \\
\hline Malva sylvestris L., Sp. P1. 2: 689 (1753) & H scap(T scap) & Subcosmop. & Native \\
\hline \multicolumn{4}{|c|}{$\begin{array}{ll} & \text { Thymelaeaceae } \\
\end{array}$} \\
\hline Daphne gnidium L., Sp. Pl. 1: 357 (1753) subsp. gnidium & P caesp & Steno-Medit.-Macaron. & Native \\
\hline Daphne laureola L., Sp. Pl. 1: 356 (1753) subsp. laureola & P caesp & Submedit.-Subatl. & Native \\
\hline \multicolumn{4}{|l|}{ Cistaceae } \\
\hline Cistus creticus L., Sp. Pl., ed. 2. 1: 738 (1762) & NP & Steno-Medit. & Native \\
\hline Cistus eriocephalus Viv., Fl. Cors. Sp. Nov. 8 (1824) & NP & Steno-Medit. & Native \\
\hline Cistus salviifolius L., Sp. P1. 1: 524 (1753) & NP & Steno-Medit. & Native \\
\hline \multicolumn{4}{|c|}{ Resedaceae } \\
\hline Reseda alba L., Sp. Pl. 1: 449 (1753) & T scap/H scap & Steno-Medit. & Native \\
\hline \multicolumn{4}{|c|}{ Brassicaceae (Cruciferae) } \\
\hline Arabis caucasica Willd., Enum. Pl. Suppl. [Willdenow] 45 (1814) & H scap & Medit.-Medit. & Native \\
\hline Arabis collina Ten., Prod. Fl. Nap. 39 (1812). & H scap & Medit.-Mont. & Native \\
\hline Arabis hirsuta (L.) Scop., Fl. Carniol., ed. 2. 2: 30 (1772) & H bienn/H scap & Euri-Medit. & Native \\
\hline $\begin{array}{l}\text { Arabis verna (L.) R. Br., in W.T. Aiton, Hort. Kew. ed. 2, 4: } \\
105(1812)\end{array}$ & H bienn/H scap & Steno-Medit. & Native \\
\hline
\end{tabular}




\begin{tabular}{|c|c|c|c|}
\hline Biscutella maritima Ten., Prod. Fl. Nap. 38 (1812) & T scap & Endem. & Native \\
\hline $\begin{array}{l}\text { Brassica rapa subsp. silvestris Janch. \& Wendelb., Kleine } \\
\text { Flora von Wien und Burgenland (1953) }\end{array}$ & T scap/H scap & Euri-Medit. & Native \\
\hline $\begin{array}{l}\text { Brassica rupestris subsp. hispida Raimondo \& P. Mazzola, } \\
\text { Lagascalia } 19 \text { (1-2): } 832 \text { (1997) }\end{array}$ & Ch suffr & Endem. Sic. & Native \\
\hline Capsella bursa-pastoris (L.) Medik., Pfl.-Gatt. 85 (1792) & H bienn & Cosmop.(Sinantrop.) & Native \\
\hline $\begin{array}{l}\text { Capsella rubella Reut., Compt.-Rend. Trav. Soc. Hallér. } \\
(1853-54) 18\end{array}$ & T scap & Euri-Medit. & Native \\
\hline Cardamine hirsuta L., Sp. Pl. 2: 655 (1753) & T scap & Cosmop. & Native \\
\hline $\begin{array}{l}\text { Diplotaxis erucoides (L.) DC., Syst. Nat. [Candolle] 2: } 631 \\
\text { (1821) }\end{array}$ & T scap & W-Medit. (Steno) & Native \\
\hline Diplotaxis viminea DC., Syst. Nat. [Candolle] 2: 635 (1821) & T scap & Euri-Medit. & Native \\
\hline Draba boerhaavii H.C.Hall, Spec. Bot. [Hall] 149 (1821) & T scap & Circumbor. & Native \\
\hline Draba muralis L., Sp. Pl. 2: 642 (1753) & T scap & Circumbor. & Native \\
\hline $\begin{array}{l}\text { Isatis canescens DC., Fl. Franc. [de Candolle \& Lamarck], } \\
\text { ed. 3. 6: } 598 \text { (1815) }\end{array}$ & H bienn & C-Medit & Native \\
\hline Lepidium draba L., Sp. P1. 2: 645 (1753) & G rhiz/H scap & Medit.-Turan. & Native \\
\hline $\begin{array}{l}\text { Nasturtium officinale R.Br., in W.T.Aiton, Hort. Kew., ed. } 2 \\
\text { [W.T. Aiton] 4: } 111 \text { (1812) }\end{array}$ & H scap & Cosmop. & Native \\
\hline $\begin{array}{l}\text { Raphanus raphanistrum L., Sp. Pl. 2: } 669 \text { (1753) subsp. } \\
\text { raphanistrum }\end{array}$ & T scap & Euri-Medit. & Native \\
\hline $\begin{array}{l}\text { Rapistrum rugosum subsp. orientale (L.) Arcang., Comp. Fl. } \\
\text { Ital. [Arcangeli] } 49 \text { (1882) }\end{array}$ & T scap & Euri-Medit. & Native \\
\hline Sinapis pubescens L., Mant. Pl. 95 (1767) & Ch suffr & SW-Steno-Medit. & Native \\
\hline Sisymbrium irio L., Sp. P1. 2: 659 (1753) & T scap & Paleo-Temp. & Native \\
\hline Sisymbrium officinale (L.) Scop., Fl. Carniol., ed. 2. 2: 26 (1772) & T scap & Subcosmop. & Native \\
\hline \multicolumn{4}{|c|}{ Santalaceae } \\
\hline Osyris alba L., Sp. Pl. 2: 1022 (1753) & NP & Euri-Medit. & Native \\
\hline \multicolumn{4}{|c|}{ Polygonaceae } \\
\hline Polygonum aviculare L., Sp. Pl. 1: 362 (1753) & T rept & Cosmop. & Native \\
\hline Rumex conglomeratus Murray, Prod. Gotting. 52 (1770) & H scap & Circumbor--S-Afric. & Native \\
\hline Rumex pulcher L., Sp. Pl. 1: 336 (1753) subsp. pulcher & H scap & Euri-Medit. & Native \\
\hline Rumex thyrsoides Desf., Fl. Atlant. 1: 321 (1798) & H scap & W-Steno-Medit. & Native \\
\hline \multicolumn{4}{|c|}{ Caryophyllaceae } \\
\hline Cerastium glomeratum Thuill., Fl. Env. Paris, ed. 2. 226 (1799) & T scap & Subcosmop. & Native \\
\hline $\begin{array}{l}\text { Dianthus graminifolius C.Presl, Fl. Sicul. (Presl) 1: } 147 \\
(1826)\end{array}$ & $\begin{array}{l}\text { H scap(Ch } \\
\text { suffr })\end{array}$ & Endem. Sic. & Native \\
\hline $\begin{array}{l}\text { Dianthus rupicola Biv., Sicul. Pl. Cent. i. } 31 \text { (1806) subsp. } \\
\text { rupicola }\end{array}$ & Ch suffr & Subendem. & Native \\
\hline Dianthus siculus C.Presl, Delic. Prag. 59. (1822). & H scap & Endem. Sic. & Native \\
\hline $\begin{array}{l}\text { Herniaria nebrodensis Jan ex Nyman, Consp. Fl. Eur. 2: } 256 \\
\text { Add. (1879) }\end{array}$ & T scap & SW-Medit.-Mont. & Native \\
\hline Silene fruticosa L., Sp. Pl. 1: 417 (1753) & Ch suffr & E-Steno-Medit. & Native \\
\hline $\begin{array}{l}\text { Silene alba (Mill.) E.H.L.Krause, Deutschl. Fl. (Sturm), ed. } \\
\text { 2. 5: } 98 \text { (1901) }\end{array}$ & $\begin{array}{l}\text { H bienn }(\mathrm{H} \\
\text { scap) }\end{array}$ & Paleo-Temp. & Native \\
\hline Silene coeli-rosa (L.) Godr., Observ. inflor. Silene 42 (1847) & T scap & SW-Steno-Medit. & Native \\
\hline $\begin{array}{l}\text { Silene commutata Guss., Fl. Sicul. Prodr. 1: } 499 \text { (1827) } \\
\text { subsp. commutata }\end{array}$ & H scap & E-Medit-Mont. & Native \\
\hline Silene fuscata Link ex Brot., Fl. Lusit. 2: 187 (1805) & T scap & Steno-Medit. & Native \\
\hline Silene gallica L., Sp. Pl. 1: 417 (1753) & T scap & Subcosmop. & Native \\
\hline
\end{tabular}




\begin{tabular}{|c|c|c|c|}
\hline $\begin{array}{l}\text { Silene sicula Ucria, in Nuov. Racc. Opusc. Aut. Sicil. vi. } \\
\text { (Pl. Linn. Op. Addend. \& Secund. Linn. Syst.) } 251 \text { (1793) }\end{array}$ & Ch suffr & Endem. & Native \\
\hline $\begin{array}{l}\text { Silene vulgaris (Moench) Garcke, Fl. N. Mitt.-Deutschland, } \\
\text { ed. } 964 \text { (1869) }\end{array}$ & H scap & Subcosmop. & Native \\
\hline $\begin{array}{l}\text { Stellaria cupaniana (Jord. \& Fourr.) Beg., Nuovo Giorn. } \\
\text { Bot. Ital. ser. 2, 15: } 552 \text { (1908) }\end{array}$ & T scap & Medit.-Turan. & Native \\
\hline $\begin{array}{l}\text { Stellaria media (L.) Vill., Hist. Pl. Dauphiné (Villars) 3(2): } \\
615 \text { (1789) }\end{array}$ & T rept/H bienn & Cosmop. & Native \\
\hline $\begin{array}{l}\text { Stellaria neglecta Weihe in M. J. Bluff \& C.A. Fingerhuth, } \\
\text { Comp. Fl. German. 1: } 560(1825)\end{array}$ & T scap & Paleo-Temp. & Native \\
\hline Spergularia rubra J.Presl \& C.Presl, Fl. Cech. 94 (1819) & $\begin{array}{l}\text { Ch suffr } \\
\text { (T scap) }\end{array}$ & Subcosmop.-Temp. & Native \\
\hline \multicolumn{4}{|c|}{$\begin{array}{ll}\text { Cornaceae } \\
\end{array}$} \\
\hline Cornus sanguinea L., Sp. Pl. 1: 117 (1753) subsp. sanguinea. & P caesp & Eurasiat.-Temp. & Native \\
\hline \multicolumn{4}{|c|}{ Primulaceae } \\
\hline Anagallis arvensis L., Sp. P1. 1: 148 (1753). & T rept & Subcosmop. & Native \\
\hline Anagallis foemina Mill., Gard. Dict., ed. 8. n. 2 (1768). & T rept & Subcosmop. & Native \\
\hline $\begin{array}{l}\text { Cyclamen hederifolium Aiton, Hort. Kew. [W. Aiton] 1: } \\
196 \text { (1789) }\end{array}$ & G bulb & N-Medit(Steno) & Native \\
\hline Cyclamen repandum Sm., Fl. Graec. Prodr. 1(1): 128 (1806) & G bulb & N-Medit. & Native \\
\hline Primula vulgaris Huds., Fl. Angl. (Hudson) 70 (1762) & $\mathrm{H}$ ros & Europ.-Caucas. & Native \\
\hline Samolus valerandi L., Sp. Pl. 1: 171 (-172) (1753) & H scap & Subcosmop. & Native \\
\hline \multicolumn{4}{|c|}{ Ericaceae } \\
\hline Erica multiflora L., Sp. Pl. 1: 355 (1753) & NP $(P$ caesp $)$ & Steno-Medit. & Native \\
\hline \multicolumn{4}{|c|}{ Rubiaceae } \\
\hline Asperula laevigata L., Mant. Pl. 38 (1767) & H scap & C-W-Steno-Medit. & Native \\
\hline Galium pallidum C.Presl, Delic. Prag. 121 (1822) & H scap & Endem. Sic. & Native \\
\hline Galium aparine L., Sp. Pl. 1: 108 (1753) & T scap & Eurasiat. & Native \\
\hline Galium lucidum All., Fl. Pedem. i. 5 (1785) subsp. lucidum & H scap & Euri-Medit. & Native \\
\hline Galium parisiense L., Sp. Pl. 1: 108 (1753) & T scap & Euri-Medit. & Native \\
\hline Galium rotundifolium L., Sp. Pl. 1: 108 (1753) & H scap & Orof.-W- Eurasiat. & Native \\
\hline $\begin{array}{l}\text { Galium verrucosum Huds., Philos. Trans. 56: } 251 \text { (1767) } \\
\text { subsp. verrucosum }\end{array}$ & T scap & Steno-Medit. & Native \\
\hline Rubia peregrina L., Sp. Pl. 1: 109 (1753) subsp. peregrina & P lian & W-C-Steno-Medit. & Native \\
\hline Sherardia arvensis L., Sp. Pl. 1: 102 (1753) & T scap & Subcosmop. & Native \\
\hline Theligonum cynocrambe L., Sp. Pl. 2: 993 (1753) & T scap & Steno-Medit. & Native \\
\hline Valantia muralis L., Sp. Pl. 2: 1051 (1753) & T scap & Steno-Medit. & Native \\
\hline \multicolumn{4}{|c|}{$\begin{array}{ll} & \text { Gentianaceae } \\
\end{array}$} \\
\hline $\begin{array}{l}\text { Blackstonia perfoliata subsp. intermedia (Ten.) Zeltner in } \\
\text { Bull. Soc. Neuchateloise Sci. Nat. 93: } 45 \text { (1970) }\end{array}$ & T scap & Steno-Medit. & Native \\
\hline $\begin{array}{l}\text { Centaurium erythraea subsp. grandiflorum (Pers.) Melderis, } \\
\text { Bot. J. Linn. Soc. } 65(2): 234 \text { (1972) }\end{array}$ & H bienn & W-Steno-Medit. & Native \\
\hline $\begin{array}{l}\text { Centaurium pulchellum (Sw.) Druce, The Flora of } \\
\text { Berkshire (1897) }\end{array}$ & T scap & Paleotemp. & Native \\
\hline \multicolumn{4}{|c|}{ Boraginaceae } \\
\hline Borago officinalis L., Sp. Pl. 1: 137 (1753) & T scap & Euri-Medit. & Native \\
\hline $\begin{array}{l}\text { Buglossoides purpurocaerulea (L.) I. M. Johnst., J. Arnold } \\
\text { Arbor. 35(1): } 44 \text { (1954) }\end{array}$ & H scap & S-Europ-Pontico & Native \\
\hline Cerinthe major L., Sp. Pl. 1: 136 (1753) & T scap & Steno-Medit. & Native \\
\hline Cynoglossum columnae Ten., Fl. Napol. 1, Prodr.: xiv. (1811) & T scap & NE-Medit.-Mont. & Native \\
\hline
\end{tabular}




\begin{tabular}{|c|c|c|c|}
\hline Cynoglossum creticum Mill., Gard. Dict., ed. 8. n. 3 (1768) & H bienn & Euri-Medit. & Native \\
\hline Echium parviflorum Moench, Methodus (Moench) 423 (1794) & $\begin{array}{l}\text { T scap } \\
\text { (H bienn) }\end{array}$ & Steno-Medit. & Native \\
\hline $\begin{array}{l}\text { Echium italicum subsp. siculum (Lacaita) Greuter \& } \\
\text { Burdet, Willdenowia 11(1): } 37 \text { (1981) }\end{array}$ & H bienn & Endem. Sic. & Native \\
\hline Echium plantagineum L., Mant. Pl. Altera 202 (1771) & T scap/H bienn & Euri-Medit. & Native \\
\hline Heliotropium europaeum L., Sp. Pl. 1: 130 (1753) & T scap & Euri-Medit.-Turan. & Native \\
\hline $\begin{array}{l}\text { Myosotis ramosissima Rochel, Oestr. Fl., ed. } 2 \text { 1: 366, } \\
\text { adnot. (1814) }\end{array}$ & T scap & Europ.-W-Asiat. & Native \\
\hline $\begin{array}{l}\text { Symphytum gussonei F.W.Schultz, in Arch. Fl. (1874) p. lviii; } \\
\text { et in Flora, lviii. (1875) } 218\end{array}$ & G rhiz & Endem. Sic. & Native \\
\hline \multicolumn{4}{|l|}{$\begin{array}{ll}\text { Convolvulaceae } \\
\end{array}$} \\
\hline $\begin{array}{l}\text { Calystegia silvatica Griseb., Spicilegium Florae Rumelicae } \\
\text { et Bithynicae (1844) }\end{array}$ & H scand & SE-Europ. & Native \\
\hline Convolvulus althaeoides L., Sp. Pl. 1: 156 (1753) & H scand & W-Steno-Medit. & Native \\
\hline Convolvulus arvensis L., Sp. Pl. 1: 153 (1753) & G rhiz & Cosmop. & Native \\
\hline Convolvulus cantabrica L., Sp. Pl. 1: 158 (1753) & H scap & Euri-medit. & Native \\
\hline $\begin{array}{l}\text { Convolvulus meonanthus Hoffmanns. \& Link, Fl. Portug. } \\
\text { [Hoffmannsegg] 1: } 369\end{array}$ & T scap & W-Steno-Medit. & Native \\
\hline $\begin{array}{l}\text { Convolvulus tricolor subsp. cupanianus (Tod.) Cavara \& } \\
\text { Grande in Boll. Soc. Bot. Ital. 1925: } 104 \text { (1925) }\end{array}$ & T scap & W-Steno-Medit. & Native \\
\hline Cuscuta epithymum L., Syst. Veg., ed. 13. 140 (1774) & T par & Eurasiat.-Temp. & Native \\
\hline \multicolumn{4}{|c|}{ Solanaceae } \\
\hline Mandragora autumnalis Bertol., Elench. 6; Spreng. Syst. i. 699 & $\mathrm{G}$ rhiz/H ros & Steno-Medit. & Native \\
\hline Solanum nigrum L., Sp. Pl.: 186 (1753) subsp. nigrum & T scap & Cosmop.(Sinantr.) & Native \\
\hline $\begin{array}{l}\text { Solanum villosum Mill., Gard. Dict., ed. 8. ed. 8, art. } \\
\text { Solanum no. } 2 \text { (1768) subsp. villosum }\end{array}$ & T scap & Euri-Medit. & Native \\
\hline \multicolumn{4}{|l|}{$\begin{array}{ll} & \text { Oleaceae } \\
\end{array}$} \\
\hline Fraxinus angustifolia Vahl, Enum. Pl. [Vahl] 1: 52 (1804) & P scap & SE-Europ.(Pontico) & Native \\
\hline Fraxinus ornus L., Sp. P1. 2: 1057 (1753) & P caesp/P scap & Euri-Medit.-Pontico & Native \\
\hline Phillyrea latifolia L., Sp. Pl. 1: 8 (1753) & P caesp/P scap & Steno-Medit. & Native \\
\hline \multicolumn{4}{|c|}{$\begin{array}{ll} & \text { Plantaginaceae } \\
\end{array}$} \\
\hline Antirrhinum siculum Mill., Gard. Dict., ed. 8. n. 6. 1768 & Ch frut & Subendem. & Native \\
\hline $\begin{array}{l}\text { Cymbalaria pubescens (C.Presl) Cufod., Bot. Not. 1947, } \\
148 \text { (1947) }\end{array}$ & $\mathrm{H} \mathrm{dec}$ & Endem. Sic. & Native \\
\hline $\begin{array}{l}\text { Kickxia spuria Dumort., Fl. Belg. (Dumortier) } 35 \text { (1827) } \\
\text { (1827) subsp. spuria }\end{array}$ & T scap & Eurasiat. & Native \\
\hline $\begin{array}{l}\text { Linaria multicaulis (L.) Mill., Gard. Dict., ed. 7. n. } 7 \text { subsp. } \\
\text { multicaulis }\end{array}$ & H scap & Endem. & Native \\
\hline $\begin{array}{l}\text { Linaria purpurea (L.) Mill., Gard. Dict., ed. 8. [unpaged] } \\
\text { Linaria no. } 5 \text { (1768) }\end{array}$ & H scap & Endem. & Native \\
\hline Misopates orontium (L.) Raf., Autik. Bot. 158 (1840) & T scap & Euri-Medit. & Native \\
\hline Plantago coronopus L., Sp. Pl. 1: 115 (1753) subsp. coronopus & $\begin{array}{c}\text { T scap/H } \\
\text { bienn/H ros }\end{array}$ & Euri-Medit. & Native \\
\hline Plantago lagopus L., Sp. Pl. 1: 114 (1753) & T scap & Steno-Medit. & Native \\
\hline Plantago lanceolata L., Sp. Pl. 1: 113 (1753) & $\mathrm{H}$ ros & Cosmop. & Native \\
\hline Plantago major L., Sp. Pl. 1: 112 (-113) (1753) subsp. major & $\mathrm{H}$ ros & Subcosmop. & Native \\
\hline Plantago serraria L., Syst. Nat., ed. 10. 2: 896 (1759) & H ros & Steno-Medit. & Native \\
\hline Veronica anagallis-aquatica L., Sp. Pl. 1: 12 (1753) & $\begin{array}{l}\text { H scap } \\
\text { (T scap) }\end{array}$ & Cosmop. & Native \\
\hline
\end{tabular}




\begin{tabular}{|c|c|c|c|}
\hline Veronica beccabunga L., Sp. Pl. 1: 12 (1753) & H rept & Eurasiat. & Native \\
\hline $\begin{array}{l}\text { Veronica cymbalaria Bodard, Mem. Veronique Cymbalaire } \\
3 \text { (1798) }\end{array}$ & T scap & Euri-Medit. & Native \\
\hline \multicolumn{4}{|l|}{$\begin{array}{ll} & \text { Scrophulariaceae } \\
\end{array}$} \\
\hline Scrophularia auriculata L., Sp. Pl. 2: 620 (1753). & H scap & Subatl. & Native \\
\hline $\begin{array}{l}\text { Scrophularia canina subsp. bicolor (Sm. in Sibth. \& Sm.) } \\
\text { Greuter in Boissiera 13: } 109 \text { (1967) }\end{array}$ & H scap & Euri-Medit. & Native \\
\hline Scrophularia peregrina L., Sp. P1. 2: 621 (1753) & T scap & Steno-Medit. & Native \\
\hline Verbascum creticum (L.) Cav., Elench. Pl. Horti Matr. 39 (1803) & H bienn & W-Steno-Medit. & Native \\
\hline Verbascum sinuatum L., Sp. Pl. 1: 178 (1753) & H bienn & Euri-Medit. & Native \\
\hline \multicolumn{4}{|c|}{ Lamiaceae (Labiatae) } \\
\hline Ajuga orientalis L., Sp. Pl. 2: 561 (1753) & H scap & E-Medit.-Mont. & Native \\
\hline Calamintha foliosa Opiz, Naturalientausch iii. (1823) [20]. & H scap & (Euri) Medit.-Mont. & Native \\
\hline $\begin{array}{l}\text { Clinopodium vulgare subsp. arundanum (Boiss.) Nyman, } \\
\text { Consp. Fl. Eur. } 587 \text { (1881) }\end{array}$ & H scap & Circumbor. & Native \\
\hline Lamium amplexicaule L., Sp. Pl. 2: 579 (1753) & T scap & Paleo-Temp. & Native \\
\hline Lamium bifidum Cirillo, Pl. Rar. Neapol. Fasc. i. 22. t. 7 (1788) & T scap & Steno-Medit. & Native \\
\hline Lamium flexuosum Ten., Fl. Napol. 2: 19, t. 52 (1820) & H scap & NW-Medit.-Mont. & Native \\
\hline Melittis albida Guss., Fl. Sicul. Prodr. 2: 140 (1828) & H scap & Medit.-Mont. & Native \\
\hline Mentha pulegium L., Sp. Pl. 2: 577 (1753) & H scap & Subcosmop. & Native \\
\hline Mentha suaveolens Ehrh., Beitr. Naturk. [Ehrhart] 7: 149 (1792). & H scap & Euri-Medit. & Native \\
\hline Micromeria canescens Benth., Labiat. Gen. Spec. 376 (1834) & Ch suffr & Endem. & Native \\
\hline $\begin{array}{l}\text { Micromeria fruticulosa (Bertol.) Šilic, Monogr. } \\
\text { Satureja Fl. Jugusl.: 256. } 1979\end{array}$ & Ch suffr & Endem. & Native \\
\hline $\begin{array}{l}\text { Micromeria graeca subsp. longiflora (C.Presl) } \\
\text { Nyman, Consp. Fl. Eur. } 590 \text { (1881) }\end{array}$ & Ch suffr & Endem. & Native \\
\hline $\begin{array}{l}\text { Micromeria graeca (L.) Benth. ex Rchb., Fl. Germ. } \\
83 \text { Excurs.: } 311 \text { (1831) subsp. graeca }\end{array}$ & Ch suffr & Steno-Medit. & Native \\
\hline Micromeria juliana Benth., Labiat. Gen. Spec. 373 (1834) & Ch suffr & Steno-Medit. & Native \\
\hline Micromeria nervosa Benth., Labiat. Gen. Spec. 376 (1834) & Ch suffr & S-Steno-Medit. & Native \\
\hline Origanum heracleoticum L., Sp. Pl. 2: 589 (1753) & H scap & SE-Steno-Medit. & Native \\
\hline Prunella vulgaris L., Sp. P1. 2: 600 (1753) & H scap & Circumbor. & Native \\
\hline Salvia clandestina L., Sp. Pl., ed. 2. 1: 36 (1762) & H scap & Steno-Medit. & Native \\
\hline $\begin{array}{l}\text { Scutellaria columnae subsp. gussonei (Ten.) } \\
\text { Nyman, Consp. F1. Eur. } 573 \text { (1881) }\end{array}$ & H scap & Endem. & Native \\
\hline Scutellaria rubicunda Hornem., Hort. Bot. Hafn. 2: 968 (1815) & H scap & Endem. & Native \\
\hline Sideritis romana L., Sp. Pl. 2: 575 (1753) & T scap & Steno-Medit. & Native \\
\hline $\begin{array}{l}\text { Stachys ocymastrum Briq., Nat. Pflanzenfam. Nachtr. } \\
\text { [Engler \& Prantl] I. } 291\end{array}$ & T scap & W-Steno-Medit. & Native \\
\hline Teucrium flavum L., Sp. Pl. 2: 565 (1753) subsp. flavum & Ch suffr(NP) & Steno-Medit. & Native \\
\hline $\begin{array}{l}\text { Teucrium scordium subspec. scordioides (Schreb.) } \\
\text { Arcang., Comp. Fl. Ital. [Arcangeli] } 559(1882)\end{array}$ & H scap & Europ.-Caucas. & Native \\
\hline Thymbra capitata (L.) Cav., Elench. Pl. Horti Matr. 37 (1803) & Ch frut & Steno-Medit. & Native \\
\hline Thymus spinulosus Ten., Prod. Fl. Nap. p. xxx v & Ch rept & Endem. & Native \\
\hline \multicolumn{4}{|c|}{ Orobanchaceae } \\
\hline Bartsia trixago L., Sp. P1. 2: 602 (1753) & T scap & Euri-Medit. & Native \\
\hline $\begin{array}{l}\text { Odontites bocconei (Guss.) Walp., Repert. Bot. Syst. } \\
\text { (Walpers) 3: 400. (1844) }\end{array}$ & Ch frut & Endem. Sic. & Native \\
\hline $\begin{array}{l}\text { Odontites rigidifolius Benth., Prodr. [A. P. de Candolle] 10: } \\
550 \text { (1846) }\end{array}$ & T scap & Subendem. & Native \\
\hline
\end{tabular}




\begin{tabular}{|c|c|c|c|}
\hline Orobanche amethystea Thuill., Fl. Par. ed. II. i. 317 (1800) & T par & Submedit.-Subatl & Native \\
\hline Orobanche crenata Forssk., Fl. Aegypt.-Arab. 113. (1775) & T par & Steno-Medit. & Native \\
\hline Orobanche hederae Duby, Bot. Gall., pars prima 350 (1828) & T par & Euri-Medit. & Native \\
\hline $\begin{array}{l}\text { Parentucellia latifolia (L.) Caruel, Fl. Ital. (Parlatore) 6: } \\
480 \text { (1885) }\end{array}$ & T scap & Euri-Medit. & Native \\
\hline Parentucellia viscosa Caruel, Fl. Ital. (Parlatore) 6(2): 482 (1885) & T scap & Medit-Atl. & Native \\
\hline \multicolumn{4}{|c|}{ Acanthaceae } \\
\hline Acanthus mollis L., Sp. P1. 2: 639 (1753) & H scap & W-Steno-Medit. & Cryptogenic \\
\hline \multicolumn{4}{|c|}{ Verbenaceae } \\
\hline Verbena officinalis L., Sp. Pl. 1: 20 (-21) (1753) & H scap & Paleo-Temp. & Native \\
\hline \multicolumn{4}{|c|}{ Aquifoliaceae } \\
\hline Ilex aquifolium L., Sp. Pl. 1: 125 (1753) & P caesp/P scap & Submedit.-Subatl. & Native \\
\hline \multicolumn{4}{|c|}{ Campanulaceae } \\
\hline Campanula erinus L., Sp. Pl. 1: 169 (1753) & T scap & Steno-Medit. & Native \\
\hline $\begin{array}{l}\text { Legousia hybrida Delarbre, Fl. Auvergne (Delarbre) ed. 2, } \\
47 \text { (1800) }\end{array}$ & T scap & Medit.-Atl. (Euri) & Native \\
\hline \multicolumn{4}{|c|}{$\begin{array}{ll} & \text { Asteraceae (Compositae) } \\
\end{array}$} \\
\hline $\begin{array}{l}\text { Achillea ligustica All., Auct. Syn. Meth. Stirp. Hort. Regii } \\
\text { Taur. } 17 \text { (1773) }\end{array}$ & H scap & W-Steno-Medit. & Native \\
\hline $\begin{array}{l}\text { Anacyclus clavatus (Desf.) Pers., Syn. Pl. [Persoon] 2(2): } \\
465 \text { (1807) }\end{array}$ & T scap & Steno-Medit. & Native \\
\hline $\begin{array}{l}\text { Anthemis arvensis subsp. sphacelata (C.Presl) R.Fernandes, } \\
\text { Bot. J. Linn. Soc. 70(1): } 12 \text { (1975) }\end{array}$ & T scap & Endem. & Native \\
\hline $\begin{array}{l}\text { Anthemis cretica subsp. columnae (Ten.) Franzén, Willdenowia } \\
\text { 16(1): } 40 \text { (1986) }\end{array}$ & H scap & Medit.-Mont. & Native \\
\hline $\begin{array}{l}\text { Anthemis cupaniana Tod. ex Nyman, Consp. Fl. Eur. 2: } \\
360 \text { (1879) }\end{array}$ & Ch suffr & Endem. Sic. & Native \\
\hline Bellis perennis L., Sp. Pl. 2: 886 (1753) & $\mathrm{H}$ ros & Europ.-Caucas. & Native \\
\hline Bellis sylvestris Cirillo, Pl. Rar. Neapol. 2: 22, t. 4 (1792) & $\mathrm{H}$ ros & Steno-Medit. & Native \\
\hline Calendula arvensis L., Sp. P1., ed. 2. 2: 1303 (1763) & $\begin{array}{l}\text { T scap } \\
\text { (H bienn) }\end{array}$ & Euri-Medit. & Native \\
\hline $\begin{array}{l}\text { Calendula suffruticosa subsp. fulgida (Raf.) } \\
\text { Guadagno, Nuovo Giorn. Bot. Ital. 29: } 64 \text { (1922) }\end{array}$ & Ch suffr & Subendem. & Native \\
\hline Carlina gummifera Less., Syn. Gen. Compos. 12 (1832) & $\mathrm{H}$ ros & Steno-Medit. & Native \\
\hline Carlina lanata L., Sp. Pl. 2: 828 (1753) & T scap & Steno-Medit. & Native \\
\hline $\begin{array}{l}\text { Carlina sicula Ten., Cat. Hort. Neapol. } 1813 \text { App. (ed. alt.) } \\
\text { (1819) }\end{array}$ & H scap & Subendem. & Native \\
\hline Carthamus caeruleus L., Sp. Pl. 2: 830 (1753) & H scap & Steno-Medit. & Native \\
\hline Carthamus lanatus L., Sp. Pl. 2: 830 (1753) & T scap & Euri-Medit. & Native \\
\hline $\begin{array}{l}\text { Centaurea busambarensis Guss., Fl. Sicul. Syn. 2(2): } \\
873 \text { (1845) }\end{array}$ & H scap & Endem. Sic. & Native \\
\hline Centaurea calcitrapa L., Sp. Pl. 2: 917 (1753) & H bienn & Subcosmop. & Native \\
\hline Centaurea sicula L., Sp. Pl. 2: 918 (1753) & H bienn & SW-Steno-Medit. & Native \\
\hline $\begin{array}{l}\text { Centaurea solstitialis subsp. schouwii (DC.) } \\
\text { Dostál, Bot. J. Linn. Soc. 71(3): } 204 \text { (1976) }\end{array}$ & H bienn & Subendem. & Native \\
\hline $\begin{array}{l}\text { Chamaemelum fuscatum (Brot.) Vasc., Anais Inst. } \\
\text { Vinho Porto No. 20, } 276 \text { (1966) }\end{array}$ & T scap & (Steno) W-Medit. & Native \\
\hline Cichorium intybus L., Sp. P1. 2: 813 (1753) subsp. intybus. & H scap & Cosmop. & Native \\
\hline $\begin{array}{l}\text { Cirsium creticum subsp. triumfetti (Lacaita) Werner, Bot. J. } \\
\text { Linn. Soc. } 70(1): 19 \text { (1975) }\end{array}$ & $\mathrm{H}$ bienn & Subendem. & Native \\
\hline
\end{tabular}




\begin{tabular}{|c|c|c|c|}
\hline $\begin{array}{l}\text { Cirsium scabrum (Poir.) Bonnet \& Barratte, Expl. Sci. } \\
\text { Tunisie, Cat. Pl. } 238 \text { (1896) }\end{array}$ & H scap & W-Steno-Medit. & Native \\
\hline Cirsium vulgare (Savi) Ten., Fl. Nop. 5: 209 & $\begin{array}{l}\text { H bienn } \\
\text { (T scap) }\end{array}$ & Subcosmop. & Native \\
\hline Chondrilla juncea L., Sp. Pl. 2: 796 (1753) & H scap & $\begin{array}{l}\text { Euri-Medit.-S-Siber. } \\
\quad \text { (Subpontico) }\end{array}$ & Native \\
\hline $\begin{array}{l}\text { Crepis bivonana Soldano \& F.Conti, Annot. Checkl. Italian } \\
\text { Vasc. F1. } 17 \text { (2005) }\end{array}$ & T scap/H bienn & Endem. Sic. & Native \\
\hline $\begin{array}{l}\text { Crepis vesicaria subsp. hyemalis (Biv.) Babc., Univ. Calif. } \\
\text { Publ. Bot. 19: } 404 \text { (1941) }\end{array}$ & T scap & Endem. & Native \\
\hline $\begin{array}{l}\text { Crepis vesicaria subsp. taraxacifolia (Thuill.) Thell., Fl. } \\
\text { Schweiz (Schinz), ed. 3. 1: } 594 \text { (1909) }\end{array}$ & T scap & Submedit.-Subatl. & Native \\
\hline Crupina crupinastrum (Moris) Vis., Fl. Dalmat. 2: 42 (1847) & T scap & Steno-Medit. & Native \\
\hline $\begin{array}{l}\text { Cynara cardunculus L., Sp. P1. 2: } 827 \text { (1753) subsp. } \\
\text { cardunculus }\end{array}$ & H scap & Steno-Medit. & Native \\
\hline $\begin{array}{l}\text { Dittrichia viscosa (L.) Greuter, Exsicc. Genav. Conserv. Bot. } \\
\text { Distrib. Fasc. 4: } 71 \text { (1973) }\end{array}$ & H scap & Euri-Medit. & Native \\
\hline Erigeron canadensis L., Sp. Pl. 2: 863 (1753) & T scap & N-America & $\begin{array}{l}\text { Naturalized } \\
\quad \text { alien }\end{array}$ \\
\hline Eupatorium cannabinum L., Sp. Pl. 2: 838 (1753) & H scap & Paleo.-Temp. & Native \\
\hline Filago pygmaea L., Sp. Pl. 2: 927 (1753) & T rept & Steno-Medit. & Native \\
\hline $\begin{array}{l}\text { Galactites tomentosus Moench, Methodus (Moench) } 558 \\
\text { (1794) }\end{array}$ & H bienn & Steno-Medit. & Native \\
\hline $\begin{array}{l}\text { Hedypnois rhagadioloides (L.) F.W. Schmidt, Samml. } \\
\text { Phys.-Oekon. Aufsatze 1: } 279 \text { (1795) subsp. rhagadioloides }\end{array}$ & T scap & Steno-Medit. & Native \\
\hline $\begin{array}{l}\text { Hedypnois rhagadioloides subsp. tubaeformis (Ten.) Hayek, } \\
\text { Repert. Spec. Nov. Regni Veg. Beih. 30(2): } 807.1931\end{array}$ & T scap & Steno-Medit. & Native \\
\hline $\begin{array}{l}\text { Helminthotheca aculeata (Vahl) Lack, Taxon } 24 \text { (1): } 111 . \\
(1975)\end{array}$ & H scap & W-Steno-Medit. & Native \\
\hline $\begin{array}{l}\text { Helminthotheca echioides (L.) Holub, Folia Geobot. Phytotax. } \\
\text { 8(2): } 176 \text { (1973) }\end{array}$ & H scap & E-Euri-Medit. & Native \\
\hline Hyoseris radiata L., Sp. Pl. 2: 808 (1753) & $\mathrm{H}$ ros & Steno-Medit. & Native \\
\hline Hyoseris scabra L., Sp. P1. 2: 809 (1753) & $\mathrm{T}$ ros & Steno-Medit. & Native \\
\hline Hypochaeris achyrophorus L., Sp. Pl. 2: 810 (1753) & T scap & Steno-Medit. & Native \\
\hline $\begin{array}{l}\text { Hypochaeris cretensis (L.) Bory \& Chaub., Expéd. Sci. Morée, } \\
\text { Bot. } 237,356(1832)\end{array}$ & H scap & C-Medit.-Mont. & Native \\
\hline $\begin{array}{l}\text { Hypochaeris laevigata (L.) Ces. \& Pass. \& Gibelli } \\
\text { Gibelli, Comp. Fl. Ital. [Cesati] Fas. 18: } 415 \text { (1878) }\end{array}$ & H ros & SW-Medit.-Mont. & Native \\
\hline Hypochaeris radicata L., Sp. Pl. 2: 811 (1753) & $\mathrm{H}$ ros & Europ.-Caucas. & Native \\
\hline $\begin{array}{l}\text { Jacobaea erratica (Bertol.) Fourr., Ann. Soc. Linn. Lyon sér. } \\
\text { 2, 16: } 404 \text { (1868) }\end{array}$ & H bienn & Euri-Medit. & Native \\
\hline $\begin{array}{l}\text { Lactuca viminea (L.) J.Presl \& C.Presl, Fl. Cech. } 160 \text { (1819) } \\
\text { subsp. viminea }\end{array}$ & H bienn & Euri-Medit. & Native \\
\hline $\begin{array}{l}\text { Lactuca muralis (L.) Gaertn., Fruct. Sem. Pl. 2(3): t. } 158 \\
\text { (1791) }\end{array}$ & H scap & Europ.-Caucas. & Native \\
\hline Leontodon tuberosus L., Sp. Pl. 2: 799 (1753) & $\mathrm{H}$ ros & Steno-Medit. & Native \\
\hline $\begin{array}{l}\text { Onopordum illyricum subsp. cardunculus (Boiss.) Arenes } \\
\text { Notul. System. (Paris) 10: 226 (1942). }\end{array}$ & H bienn/H scap & E-Steno-Medit. & Native \\
\hline $\begin{array}{l}\text { Pallenis spinosa (L.) Cass., Dict. Sci. Nat., ed. 2. [F. Cuvier] } \\
\text { 37: } 276 \text { (1825) subsp. spinosa. }\end{array}$ & H scap & Euri-Medit. & Native \\
\hline $\begin{array}{l}\text { Phagnalon rupestre (L.) DC., Prodr. [A. P. de Candolle] 5: } \\
396 \text { (1836) subsp. rupestre. }\end{array}$ & Ch suffr & SW-Medit.(Steno) & Native \\
\hline $\begin{array}{l}\text { Phagnalon saxatile (L.) Cass., Bull. Sci. Soc. Philom. Paris } \\
174 \text { (1819) }\end{array}$ & Ch suffr & W-Medit. (Steno) & Native \\
\hline
\end{tabular}




\begin{tabular}{|c|c|c|c|}
\hline $\begin{array}{l}\text { Picris hieracioides subsp. spinulosa (Bertol. ex Guss.) Arcang., } \\
\text { Comp. Fl. Ital. [Arcangeli] } 418 \text { (1882) }\end{array}$ & H bienn/H scap & N-Euri-Medit. & Native \\
\hline $\begin{array}{l}\text { Podospermum canum C.A.Mey., Verz. Pfl. Casp. Meer. } \\
\text { (C.A. von Meyer). } 62 \text { (1831) }\end{array}$ & H scap & Eurasiat. & Native \\
\hline $\begin{array}{l}\text { Pulicaria dysenterica (L.) Bernh., Syst. Verz. (Bernhardi) } \\
153 \text { (1800) }\end{array}$ & H scap & Euri-Medit. & Native \\
\hline Pulicaria odora (L.) Rchb., Fl. Germ. Excurs. 239 & H scap & Euri-Medit. & Native \\
\hline $\begin{array}{l}\text { Reichardia intermedia (Sch. Bip.) Samp. in Bol. Soc. Brot. } \\
\text { 24: } 68 \text { (1909) }\end{array}$ & H scap & Saharo-Sind. & Native \\
\hline Reichardia picroides (L.) Roth, Bot. Abh. Beobacht. 35 (1787) & H scap & Euri-Medit. & Native \\
\hline Scolymus grandiflorus Desf., Fl. Atlant. 2: 240 (1799) & H scap & W-Steno-Medit. & Native \\
\hline $\begin{array}{l}\text { Scorzonera villosa subsp. columnae (Guss.) Nym., Consp. } \\
\text { Fl. Eur. } 465 \text { (1879) }\end{array}$ & G rhiz & Endem. & Native \\
\hline $\begin{array}{l}\text { Senecio squalidus subsp. rupestris (Waldst. \& Kit.) Greuter, } \\
\text { Willdenowia 35: } 238(2005)\end{array}$ & H scap & Euri-Medit. & Native \\
\hline Senecio vulgaris L., Sp. Pl. 2: 867 (1753) & T scap & Cosmop. & Native \\
\hline $\begin{array}{l}\text { Silybum marianum (L.) Gaertn., Fruct. Sem. Pl. 2(3):378, } \\
\text { t. 168. (1791). }\end{array}$ & H bienn & Medit.-Turan. & Native \\
\hline $\begin{array}{l}\text { Sonchus asper (L.) Hill, Herb. Brit. [Hill] 1: } 47 \text { (1769) } \\
\text { subsp. asper }\end{array}$ & T scap & Subcosmop. & Native \\
\hline Sonchus oleraceus L., Sp. Pl. 2: 794 (1753) & T scap & Subcosmop. & Native \\
\hline Sonchus tenerrimus L., Sp. Pl. 2: 794 (1753) & T scap & Steno-Medit. & Native \\
\hline $\begin{array}{l}\text { Symphyotrichum squamatum (Spreng.) G. L. Nesom, } \\
\text { Phytologia 77(3): } 292 \text { (1995) }\end{array}$ & T scap/H scap & S-America & $\begin{array}{l}\text { Naturalized } \\
\text { alien }\end{array}$ \\
\hline $\begin{array}{l}\text { Tolpis virgata (Desf.) Bertol., Mem. Soc. Med. Emul. Genova } \\
\text { 2: } 135 \text { (1803) }\end{array}$ & T scap & Steno-Medit. & Native \\
\hline $\begin{array}{l}\text { Tragopogon porrifolius subsp. cupanii (Guss. ex DC.) I. } \\
\text { Richardson, Bot. J. Linn. Soc. } 71(4): 270 \text { (1976) }\end{array}$ & H bienn/T scap & Endem. & Native \\
\hline $\begin{array}{l}\text { Urospermum dalechampii (L.) Scop. ex F. W. Schmidt, } \\
\text { Samml. Phys.-Oekon. Aufsatze 1: } 276 \text { (1795) }\end{array}$ & H scap & C-W-Euri-Medit. & Native \\
\hline $\begin{array}{l}\text { Urospermum picroides (L.) Scop. ex F. W. Schmidt, Samml. } \\
\text { Phys.-Oekon. Aufsatze 1: } 275 \text { (1795) }\end{array}$ & T scap & Euri-Medit. & Native \\
\hline Xanthium spinosum L., Sp. Pl. 2: 987 (1753) & T scap & S-America & $\begin{array}{l}\text { Naturalized } \\
\text { alien }\end{array}$ \\
\hline \multicolumn{4}{|c|}{ Viburnaceae } \\
\hline Sambucus ebulus L., Sp. Pl. 1: 269 (1753) & G rhiz(H scap) & Euri-Medit. & Native \\
\hline \multicolumn{4}{|l|}{ Caprifoliaceae } \\
\hline Lonicera etrusca Santi, Viagg. Mont. i. 113 (1795) & $\mathrm{P}$ lian( $\mathrm{P}$ caesp $)$ & Euri-Medit. & Native \\
\hline Lonicera implexa Aiton, Hort. Kew. [W. Aiton] i. 231 (1789) & P lian(P caesp. $)$ & Steno-Medit. & Native \\
\hline \multicolumn{4}{|c|}{ Dipsacaceae } \\
\hline Dipsacus fullonum L., Sp. Pl. 1: 97 (1753). & H bienn & Euri-Medit. & Native \\
\hline $\begin{array}{l}\text { Sixalix atropurpurea subsp. grandiflora (Scop.) Soldano } \\
\text { \& F. Conti, Annot. Checkl. Italian Vasc. Fl. } 22 \text { (2005) }\end{array}$ & $\begin{array}{l}\text { H bienn/ } \\
\text { T scap }\end{array}$ & Steno-Medit. & Native \\
\hline \multicolumn{4}{|l|}{$\begin{array}{ll}2 & \text { Valerianaceae }\end{array}$} \\
\hline $\begin{array}{l}\text { Centranthus ruber (L.) DC., Fl. Franc. [de Candolle } \\
\text { \& Lamarck], ed. 3. 4: } 239 \text { (1805) }\end{array}$ & Ch suffr & Steno-Medit. & Native \\
\hline $\begin{array}{l}\text { Fedia graciliflora Fisch. \& C.A.Mey., Index Seminum } \\
\text { [St. Petersburg (Petropolitanus)] 6: } 59 \text { (1840) subsp. graciliflora }\end{array}$ & T scap & S-C-Steno-Medit. & Native \\
\hline Valerianella carinata Loisel., Not. Fl. France 149 (1810) & T scap & E-Euri-Medit. & Native \\
\hline $\begin{array}{l}\text { Valerianella eriocarpa Desv., J. Bot. (Desvaux) 2: } \\
314, \text { t. } 11, \text { f. } 2 \text { (1809) }\end{array}$ & T scap & Steno-Medit. & Native \\
\hline
\end{tabular}




\begin{tabular}{|c|c|c|c|}
\hline Hedera helix L., Sp. P1. 1: 202 (1753) & P lian & Submedit.-Subatl. & Native \\
\hline \multicolumn{4}{|c|}{ Apiaceae (Umbelliferae) } \\
\hline Athamanta sicula L., Sp. Pl. 1: 244 (1753) & H scap & SW-Steno-Medit. & Native \\
\hline Bupleurum odontites L., Sp. Pl. 1: 237 (1753) & T scap & SE-Steno-Medit. & Native \\
\hline Bupleurum tenuissimum L., Sp. Pl. 1: 238 (1753) & T scap & Euri-Medit. & Native \\
\hline Daucus carota L., Sp. Pl. 1: 242 (1753) subsp. carota & $\begin{array}{l}\text { H bienn } \\
\text { (T scap) }\end{array}$ & Subcosmop. & Native \\
\hline $\begin{array}{l}\text { Elaeoselinum meoides W.D.J. Koch ex DC., Prodr. [A. P. } \\
\text { de Candolle] 4: } 215 \text { (1830) }\end{array}$ & H scap & Steno-Medit. & Native \\
\hline Eryngium campestre L., Sp. Pl. 1: 233 (1753) & H scap & Euri-Medit. & Native \\
\hline Eryngium crinitum C.Presl, Delic. Prag. 139 (1822) & H scap & Endem. Sic. & Native \\
\hline $\begin{array}{l}\text { Eryngium tricuspidatum subsp. bocconei (Lam.) A. Wörz, } \\
\text { Stuttgarter Beitr. Naturk., A 596: } 35 \text { (1999) }\end{array}$ & H scap & Endem. Sic. & Native \\
\hline Eryngium triquetrum Vahl, Symb. Bot. (Vahl) ii. 46. (1791). & H scap & SW-Steno-Medit. & Native \\
\hline Ferula communis L., Sp. Pl. 1: 246 (1753) & H scap & S-Medit.(Euri) & Native \\
\hline $\begin{array}{l}\text { Foeniculum vulgare subsp. piperitum (Ucria) Beg., Ann. } \\
\text { Mus. Civico Storia Nat. Genova ser. 3, 3: } 447 \text { (1904) }\end{array}$ & H scap & Steno-Medit. & Native \\
\hline $\begin{array}{l}\text { Foeniculum vulgare Mill., Gard. Dict. ed. 8: n. 1(1768) } \\
\text { subsp. vulgare }\end{array}$ & H scap & Euri-Medit. & Native \\
\hline $\begin{array}{l}\text { Helosciadium nodiflorum (L.) W.D.J.Koch, Nova Acta } \\
\text { Phys.-Med. Acad. Caes. Leop.-Carol. Nat. Cur. 12(1): } 126 \\
\text { (1824) subsp. nodiflorum }\end{array}$ & H scap & Euri-Medit. & Native \\
\hline $\begin{array}{l}\text { Katapsuxis silaifolia (Jacq.) Reduron \& Charpin \& Pimenov, } \\
\text { J. Bot. Soc. Bot. France 1: } 99 \text { (1997) }\end{array}$ & H scap & SE-Europ. & Native \\
\hline $\begin{array}{l}\text { Kundmannia sicula DC., Prodr. [A. P. de Candolle] 4: } \\
143 \text { (1830) }\end{array}$ & H scap & Steno-Medit. & Native \\
\hline $\begin{array}{l}\text { Magydaris pastinacea (Lam.) Paol., Fl. Italia [Fiori, Béguinot } \\
\text { \& Paoletti] 2: } 205 \text { (1900) }\end{array}$ & H scap & W-Steno-Medit. & Native \\
\hline Oenanthe globulosa L., Sp. Pl. 1: 255 (1753) & H scap & W-Steno-Medit. & Native \\
\hline Oenanthe pimpinelloides L., Sp. P1. 1: 255 (1753) & H scap & Medit.-Atl. & Native \\
\hline Opopanax chironium Guss., Fl. Sicul. Syn. 1: 352 (1843) & H scap & Steno-Medit. & Native \\
\hline $\begin{array}{l}\text { Physospermum verticillatum Vis., Fl. Dalmat. iii. 358, in } \\
\text { obs. (1851) }\end{array}$ & H scap & Medit.-Mont. & Native \\
\hline Pimpinella anisoides V.Brig., Nova Pimp. Spec. Diss. 11 (1802) & H scap & Endem. & Native \\
\hline Pimpinella peregrina L., Sp. Pl. 1: 264 (1753) & H bienn & Euri-Medit. & Native \\
\hline $\begin{array}{l}\text { Scandix pecten-veneris L., Sp. Pl. 1: } 256 \text { (1753) subsp. } \\
\text { pecten-veneris }\end{array}$ & T scap & Euri-Medit. & Native \\
\hline Smyrnium olusatrum L., Sp. Pl. 1: 262 (1753) & H bienn & Medit.-Atl. & Native \\
\hline $\begin{array}{l}\text { Smyrnium perfoliatum subsp. rotundifolium (Mill.) } \\
\text { Hartvig, Mountain Fl. Greece [Strid] 1: } 672 \text { (1986) }\end{array}$ & H bienn & Steno-Medit. & Native \\
\hline Thapsia garganica L., Mant. Pl. 57 (1767) & H scap & Steno-Medit. & Native \\
\hline Tordylium apulum L., Sp. Pl. 1: 239 (1753) & T scap & Steno-Medit. & Native \\
\hline Visnaga daucoides Gaertn., Fruct. Sem. Pl. i 92. t. 21 (1788) & T scap & Euri-Medit. & Native \\
\hline
\end{tabular}




\section{REFERENCES}

Badalamenti E., Cusimano D., La Mantia T., Pasta S., Romano S., Troia A. \& Ilardi V., 2018. The ongoing naturalisation of Eucalyptus spp. in the Mediterranean Basin: new threats to native species and habitats. Australian Forest., 81: 239-249. https://doi.org/10.1080/00049158.2018.1533512.

Bazan G., Marino P., Guarino R., Domina G. \& Schicchi R., 2015. Bioclimatology and vegetation series in Sicily: a geostatistical approach. Annales Botanici Fennici, 52: 1-18. DOI: 10.5735/085.052.0202.

Brullo S., Minissale P. \& Spampinato G., 1995. Considerazioni fitogeografiche sulla flora della Sicilia. Ecologia Mediterranea, 21 (1/2): 99-117.

Brullo S., Pavone P. \& Salmeri C., 2015. Biosystematic researches on Allium cupani group (Amaryllidaceae) in the Mediterranean area. Flora Mediterranea, 25 (Special Issue): 209-244. https://doi.org/10.7320/FlMedit25SI.209.

Caldarella O., La Rosa A., Pasta S. \& Di Dio V., 2010. La flora vascolare della Riserva Naturale Orientata Isola delle Femmine (Sicilia nord-occidentale): aggiornamento della check-list e analisi del turnover. Il Naturalista siciliano, 34: 421-476.

Catalano R., Franchino A., Merlini S. \& Sulli A., 2000. Central western Sicily structural setting interpreted from seismic reflection profiles. Memorie della Società Geologica Italiana, 55: 5-16.

Catalano R., Basilone L., Di Maggio C., Gasparo Morticelli M., Agate M. \& Avellone G., 2013. Note illustrative della Carta Geologica d'Italia alla scala 1:50.000 del foglio 594-585 "Partinico- Mondello", con carta geologica 1:50.000 allegata. Progetto Carg, ISPRA-Regione Siciliana, 1-271 pp.

Celesti-Grapow L., Alessandrini A., Arrigoni P. V., Banfi E., Bernardo L., Bovio M., Brundu G., Cagiotti M. R., Camarda I., Carli E., Conti F., Fascetti S., Galasso G., Gubellini L., La Valva V., Lucchese F., Marchiori S., Mazzola P., Peccenini S., Poldini L., Pretto F., Prosser F., Siniscalco C., Villani M.C., Viegi L., Wilhalm T. \& Blasi C., 2009. Inventory of the non-native flora of Italy. Plant Biosystems, 143: $386-430$. https://doi.org/10.1080/11263500902722824.

Christenhusz M.J.M., Zhang X-C. \& Schneider H., 2011. A linear sequence of extant families and genera of lycophytes and ferns. Phytotaxa, 19: 7-54. https://doi.org/10.11646/phytotaxa.19.1.2.

Dia M.G., Maniscalco M. \& Raimondo F.M., 2000. Caratterizzazione della diversità forestale e briofitica dei Monti di Palermo in rapporto ad indirizzi di gestione del territorio. Quaderni di Botanica Ambientale applicata, 8 (1997): 109-125.

Drago A., 2005. Atlante climatologico della Sicilia, Se- conda edizione. Rivista Italiana di Agrometeorologia, 10: 67-83.

Fierotti G., 1997. I suoli della Sicilia. Dario Flaccovio Ed., Palermo, 359 p.

Fiori A., 1923-25. Nuova Flora Analitica d'Italia. Tipografia Ricci, Firenze.

Gianguzzi L., D’Amico A. \& Caldarella O., 2007. La flora vascolare dei Monti di Palermo. Collana Sicilia Foreste 36. Azienda Foreste Demaniali della Regione Siciliana, Palermo, 360 pp.

Gianguzzi L., D’Amico A., Caldarella O., Ottonello D. \& Romano S., 2009. La flora vascolare e lichenica della Riserva Naturale Grotta Conza (Sicilia Nordoccidentale). Il Naturalista siciliano, 33: 33-68.

Giardina G., Raimondo F.M. \& Spadaro V., 2007. A catalogue of plants growing in Sicily. Bocconea, 20: 5582.

Greuter W., Burdet H.M. \& Long G., 1984-1989. MedChecklist, A critical inventory of vascular plants of the circum-mediterranean countries. Voll. 1, 3, 4. Annuaire du Conservatoire et du Jardin botaniques de Genève, Genève.

Guarino R. \& Pasta S., 2018. Sicily: the island that didn't know to be an archipelago. Ber. Reinhold Tüxen Ges., 30: 133-148.

Gussone G., 1827-1828. Florae Siculae Prodromus. Voll. 1-2. Ex Regia Typographia, Napoli.

Gussone G., 1842-1844. Florae Siculae Synopsis. Voll. 1-2. Ex Tipis Tramater, Napoli.

Hammond H., 2013. Landed identity and the Bourbon Neapolitan State: Claude-Joseph Vernet and the politics of the siti reali'. In: Hills H. \& Calaresu M. (eds.) New Approaches to Naples, c. 1500-1800: The Power of Place. Farnham: Ashgate, pp. 121-146.

Haston E., Richardson J.E., Stevens P.F., Chase M.W. \& Harris D.J., 2009. The Linear Angiosperm Phylogeny Group (LAPG) III: a linear sequence of the families in APG III. The Journal of the Linnean Society. Botany, 161(2): 128-131. https://doi.org/10.1111/j.1095-8339.2009.01000.x.

Johns J., 1986. Nota sugli insediamenti rupestri musulmani nel territorio di S. Maria di Monreale nel dodicesimo secolo. In: Fonseca C.D. La Sicilia rupestre nel contesto delle civiltà mediterranee. Atti del VI Convegno Internazionale di studio sulla civiltà rupestre medioevale nel Mezzogiorno d'Italia (CataniaPantalica-Ispica 7-12 settembre 1981). Galatina, 227-234 pp.

Lo Giudice M., 1849. Notizie dello Stato antico e moderno dell'arcivescovado di Monreale. Stamperia e Ligatoria di F. Ruffino, Palermo.

Lojacono Pojero M., 1888-1909. Flora Sicula. Voll. 12-3, Palermo.

Marcenò C. \& Ottonello D., 1993. Osservazioni fitosociologiche su alcune leccete dei Monti di Palermo 
(con appendice floristica). Atti dell'Accademia di scienze, lettere e arti di Palermo, serie 5, 9 (1991): 123.

Marcenò C., Colombo P. \& Princiotta R., 1985. Ricerche climatologiche e botaniche sui Monti Sicani (Sicilia centro occidentale): La flora. Il Naturalista Siciliano, 8 (suppl. 1): 69-133.

Médail F. \& Quézel P., 1997. Hot-spots analysis for conservation of plant biodiversity in the Mediterranean basin. Annales of the Missouri Botanical Garden, 84: 112-127.

Parlatore F., 1845. Flora Palermitana. Società Tipografica, Firenze.

Parlatore F., 1848-1896. Flora Italiana. Voll. 1-10. Firenze.

Pasta S., 1993. Considerazioni teoriche sulle prospettive di ricerca aperte dalla geobotanica ed esempi di applicazione di questa disciplina al caso specifico dello studio della flora e della vegetazione di un settore dei Monti di Palermo. Tesi di laurea in Scienze Naturali, Università degli Studi di Palermo, Relatore prof. C. Marcenò.

Pasta S. \& Troia A., 1994. Contributo alla conoscenza della flora dei Monti di Palermo. Il Naturalista Siciliano, 18: 15-27.

Pignatti S. 1982. Flora d'Italia. Voll. 1-3. Edagricole, Bologna.
Pignatti S., 1994. Ecologia del Paesaggio. UTET, Torino, $238 \mathrm{pp}$.

Pignatti S., Guarino R. \& La Rosa M., 2017-2019. Flora d'Italia, ed. 2. Voll. 1-4. Edagricole-New Business Media, Milano-Bologna.

Raimondo F.M. \& Mazzola P., 1997. A new taxonomic arrangement in the Sicilian members of Brassica L. sect. Brassica. Lagascalia, 19: 831-838.

Raimondo F.M., Mazzola P. \& Schicchi R., 2001. Rapporti fitogeografici fra i promontori carbonatici della costa tirrenica della Sicilia. Biogeographia, 22: 6577.

Raunkiaer C., 1934. The life forms of plants and statistical plant geography. Clarendon Press, Oxford.

Rivas-Martínez S., 2004. Global Bioclimatics (Clasificación Bioclimática de la Tierra). Departamento de Biologia Vegetal II (Botanica). Universidad Complutense, Madrid.

Sessa E., 2015. Le Tenute Reali dei Borbone in Sicilia. In: Davì G. \& Mauro E. La Casina Cinese nel regio Parco della Favorita di Palermo. Centro Regionale per l'Inventario, la Catalogazione e la Documentazione, Regione Siciliana, Palermo, 135-162 pp.

Tutin T.G., Heywood V.H., Burges N.A., Moore D.M., Valentine D.H., Walters S.M. \& Webb D.A., 19641980. Flora Europaea. Voll. 1-5. Cambridge Univ. Press, Cambridge. 
. 